\title{
Eisenstein series for higher-rank groups and string theory amplitudes
}

\author{
Michael B. Green, Stephen D. Miller, Jorge G. Russo \\ And Pierre VANhove
}

\begin{abstract}
Scattering amplitudes of superstring theory are strongly constrained by the requirement that they be invariant under dualities generated by discrete subgroups, $E_{n}(\mathbb{Z})$, of simply laced Lie groups in the $E_{n}$ series $(n \leq 8)$. In particular, expanding the foursupergraviton amplitude at low energy gives a series of higher derivative corrections to Einstein's theory, with coefficients that are automorphic functions with a rich dependence on the moduli. Boundary conditions supplied by string and supergravity perturbation theory, together with a chain of relations between successive groups in the $E_{n}$ series, constrain the constant terms of these coefficients in three distinct parabolic subgroups. Using this information we are able to determine the expressions for the first two higher derivative interactions (which are BPS-protected) in terms of specific Eisenstein series. Further, we determine key features of the coefficient of the third term in the low-energy expansion of the four-supergraviton amplitude (which is also BPS-protected) in the $E_{8}$ case. This is an automorphic function that satisfies an inhomogeneous Laplace equation and has constant terms in certain parabolic subgroups that contain information about all the preceding terms.
\end{abstract}

2. Eisenstein series, parabolic subgroups and their constant terms 
2.3. Constant term formulas

2.4. Brief description of explicit computations used later in the paper

3. String theory amplitudes and their degeneration limits $\mathbf{5 6 8}$

3.1. Solutions for the coefficients $\mathcal{E}_{(0,0)}^{(D)}$ and $\mathcal{E}_{(1,0)}^{(D)}$

3.2. Bifurcation of $\mathcal{E}_{(1,0)}^{(D)}$ between $D=5$ and $D=6$

576

4. Constant terms for the solution of the $E_{8}$ inhomogeneous Laplace eigenvalue equation

5. Summary and comments 585

Acknowledgments

587

Appendix A. Tables A.1 to A.8

588

A.1. Duality groups and maximal parabolic subgroups

588

A.2. Solutions for $\mathcal{E}_{(0,0)}^{(D)}$ and $\mathcal{E}_{(1,0)}^{(D)}$ for $\operatorname{rank}-(10-D)$ duality groups

589

A.3. Constant terms for $\mathcal{E}_{(0,0)}^{(D)}$ and $\mathcal{E}_{(1,0)}^{(D)}$

References

593

\section{Introduction}

Superstring theory is highly constrained by dualities combined with supersymmetry. These constraints are particularly strong in theories with maximal supersymmetry, which can be obtained by compactification of ten-dimensional type II closed-string theories on a $d$-torus, $\mathcal{T}^{d}$, from $D=10$ dimensions to $D=10-d$, in which case the theory is invariant under discrete subgroups $E_{d+1}(\mathbb{Z})$ of the real split forms of the Lie groups $E_{d+1}$ defined in $[1,2]^{1}$

\footnotetext{
${ }^{1}$ The symbol $E_{d+1}$ will always refer to the real split forms of these groups, which are often denoted elsewhere by $E_{d+1 \mid d+1}$ or $E_{d+1(d+1)}$.
} 
One fruitful direction for investigating the nature of these constraints has been the study of terms in the low-energy expansion of string theory amplitudes that generalize the amplitudes of classical supergravity. For example, the four-supergraviton amplitude for either of the compactified type II string theories may be decomposed into the sum of the classical supergravity treelevel contribution, an analytic part and a nonanalytic part,

$$
A_{D}(s, t, u)=A^{\text {classical }}(s, t, u)+A_{D}^{\text {analytic }}(s, t, u)+A_{D}^{\text {nonan }}(s, t, u),
$$

where the Mandelstam invariants $s, t, u(s+t+u=0)$ are quadratic in the momenta of the scattering particles. ${ }^{2}$ The classical supergravity contribution that follows from the Einstein-Hilbert action can be written as

$$
A^{\text {classical }}(s, t, u)=\frac{3}{\sigma_{3}} \mathcal{R}^{4},
$$

while the analytic part has a low-energy expansion in powers of $s, t, u$, of the form

$$
A_{D}^{\text {analytic }}(s, t, u)=\sum_{p, q=0}^{\infty} \mathcal{E}_{(p, q)}^{(D)}\left(\phi_{E_{d+1} / K}\right) \sigma_{2}^{p} \sigma_{3}^{q} \mathcal{R}^{4},
$$

where $3 \leq D=10-d \leq 10, \sigma_{n}=\left(s^{n}+t^{n}+u^{n}\right)\left(\ell_{D}^{2} / 4\right)^{n}$ and $\ell_{D}$ is the $D$-dimensional Planck length. The term "supergraviton" refers to the 256 massless physical states of the maximal supergravity multiplet, which have superhelicities that enter in the generalized curvature tensor, $\mathcal{R}$. The four powers of this tensor in the kinematic factor $\mathcal{R}^{4}$ are contracted by a rank-16 tensor, which is defined in [3]. Since $s, t$ and $u$ are quadratic in momenta, a term of the form $\sigma_{2}^{p} \sigma_{3}^{q} \mathcal{R}^{4}$ contributes a term in an effective action of the form $\partial^{4 p+6 q} \mathcal{R}^{4}$ (where the derivatives are contracted into each other in a standard manner). So the infinite series of higher momentum terms translates into a series of higher derivative local interactions in an effective action that generalizes the Einstein-Hilbert action. The nonanalytic contribution, $A_{D}^{\text {nonan }}$, contains threshold singularities in $s, t, u$ which depend on the dimension, $D$. Although there is generally no unambiguous way of disentangling these from the analytic part, this issue does not affect the terms of low order that are the concern of this paper. Nevertheless, even in the simplest cases the known threshold structure provides strong constraints on the behaviour of the coefficients in (1.3) near the cusp at which the $d$-torus decompactifies to the $(d-1)$-torus [4], which will be of importance later.

\footnotetext{
${ }^{2}$ The Mandelstam invariants are $s=-\left(k_{1}+k_{2}\right)^{2}, t=-\left(k_{1}+k_{4}\right)^{2}, u=-\left(k_{1}+\right.$ $\left.k_{3}\right)^{2}$, where $k_{r}(r=1,2,3,4)$ is the null momentum of particle $r$.
} 
The duality symmetry of string theory implies that the $D$-dimensional amplitude should be invariant under the action of the duality group, $E_{d+1}(\mathbb{Z})$. As a consequence, the coefficient functions, $\mathcal{E}_{(p, q)}^{(D)}\left(\phi_{E_{d+1} / K}\right)$, in (1.3) must be automorphic functions of the symmetric space moduli, $\phi_{E_{d+1} / K}$, that parameterize the coset space $E_{d+1} / K$ appropriate to compactification on $\mathcal{T}^{d}$, where $K$ is the maximal compact subgroup of the duality group $E_{d+1}$. The list of the various duality groups is given in table A.1. These moduli-dependent coefficient functions contain a wealth of information relating perturbative and non-perturbative string theory effects.

Although the structure of generic coefficients appears to be highly intractable, the first three terms, for which $2 p+3 q \leq 3$, are expected to display simplifying features as a consequence of maximal supersymmetry. These three interactions preserve a fraction of the complete 32-component supersymmetry, and should therefore be described as "F-terms," or fractional BPS interactions. To be explicit, the interaction $\mathcal{E}_{(0,0)}^{(D)} \mathcal{R}^{4}$ is $1 / 2$-BPS, $\mathcal{E}_{(1,0)}^{(D)} \partial^{4} \mathcal{R}^{4}$ is $1 / 4$-BPS and $\mathcal{E}_{(0,1)}^{(D)} \partial^{6} \mathcal{R}^{4}$ is $1 / 8$-BPS. A BPS condition on an interaction generally implies that it is protected from receiving perturbative contributions beyond a certain order. In other words, such functions should have a finite number of power-behaved terms when expanded around their cusps. They should also have a calculable spectrum of instanton, or nonzero mode, contributions. However, it is notoriously difficult to determine the extent of the constraints imposed on systems with maximal supersymmetry due to the absence of a covariant off-shell formulation. The next term in the expansion, $\mathcal{E}_{(2,0)}^{(D)} \partial^{8} \mathcal{R}^{4}$, is expected to be non-BPS [5], and therefore not protected by supersymmetry, in which case its coefficient is likely to have an infinite number of perturbative terms (power-behaved components in its expansion around any cusp).

Although the coefficients, $\mathcal{E}_{(p, q)}^{(D)}$, have not been determined in generality, a significant amount of information has accumulated for the first three terms for the cases with $D \geq 6$ (duality groups $E_{d+1}$ with $d \leq 4$ ) [6-12], and there are various conjectures concerning the coefficient of the $\mathcal{R}^{4}$ interaction, $\mathcal{E}_{(0,0)}^{(D)}$, for higher-rank groups $[4,13-16]$. In addition, there are partial results for $2 p+3 q \leq 6$ in $D=9$ dimensions with duality group $\mathrm{SL}(2)$ [17].

The structure of the coefficients is highly constrained by a combination of string theory and M-theory input, which provides asymptotic information at various cusps in the space of moduli, together with an analysis of the constraints imposed by supersymmetry [18]. Extending this to the exceptional groups, relevant to the theory in $D=3,4,5$ dimensions, requires more sophisticated techniques, which we will develop in this paper. In 
particular, the coefficients $\mathcal{E}_{(0,0)}^{(D)}$ and $\mathcal{E}_{(1,0)}^{(D)}$ satisfy the Laplace eigenvalue equations [4],

$$
\begin{aligned}
& \left(\Delta^{(D)}-\frac{3(11-D)(D-8)}{D-2}\right) \mathcal{E}_{(0,0)}^{(D)}=6 \pi \delta_{D-8,0} \\
& \left(\Delta^{(D)}-\frac{5(12-D)(D-7)}{D-2}\right) \mathcal{E}_{(1,0)}^{(D)}=40 \zeta(2) \delta_{D-7,0}
\end{aligned}
$$

where $\Delta^{(D)}$ is the Laplace operator on the symmetric space $E_{d+1} / K$. The Kronecker $\delta$ contributions on the right-hand side of these equations arise from anomalous behaviour, indicating the presence of polar terms for specific values of $D$ for which the eigenvalues in (1.4) and (1.5) vanish. The coefficient $\mathcal{E}_{(0,1)}^{(D)}$ satisfies the inhomogeneous Laplace eigenvalue equation

$$
\left(\Delta^{(D)}-\frac{6(14-D)(D-6)}{D-2}\right) \mathcal{E}_{(0,1)}^{(D)}=-\left(\mathcal{E}_{(0,0)}^{(D)}\right)^{2}+120 \zeta(3) \delta_{D-6,0}
$$

which involves a source term on the right-hand side that is quadratic in the $\mathcal{R}^{4}$ coefficient $\mathcal{E}_{(0,0)}^{(D)}$. The origin of (1.4) to (1.6) and, in particular, the values of the eigenvalues on the left-hand sides of these equations was discussed in $[4$, Appendix $\mathrm{H}]$.

Automorphic functions of moderate growth (which we assume ours are because of physical constraints) are nearly determined by imposing boundary conditions that specify the behaviour of their constant terms in various maximal parabolic subgroups that arise at boundaries of moduli space; the only possibility ambiguity is an additive cusp form. The constant terms are zero Fourier modes with respect to integration over the unipotent radical, $N$, in the Langlands decomposition $P=M N$ of a parabolic subgroup, $P$, where $M$ is its Levi factor. The expressions for the constant terms corresponding to three particular maximal parabolic subgroups were derived explicitly in [4] for the $E_{d+1}$ with $0 \leq d \leq 4$ and for $5 \leq d \leq 7$ will be derived in this paper.

The Levi component $M$ has the form GL(1) $\times G_{d}$, where $G_{d}$ is a rank $d$ subgroup that corresponds to deleting nodes $d+1,1$ or 2 from the Dynkin in figure 1 , and is given in table A.2. Such constant terms contain a finite sum of components, of the form $\sum_{i} r^{p_{i}} \mathcal{F}_{p_{i}}^{G_{d}}$, where $p_{i}$ are rational numbers, $r$ is the parameter for the GL(1) factor defined in (2.4), and the coefficients $\mathcal{F}_{p}^{G_{d}}$ are automorphic functions for the subgroup $G_{d}(\mathbb{Z})$. However, for the three subgroups appropriate to the string theory and supergravity calculations, the coefficients are expected to be maximal parabolic Eisenstein series. 


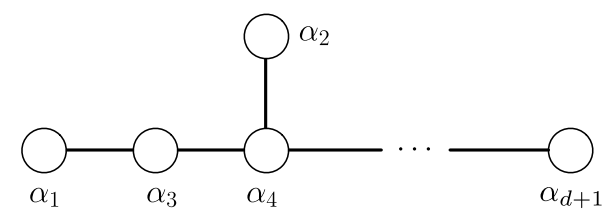

Figure 1: The Dynkin diagrams of the U-duality groups real split form of rank $d+1$ Lie group $E_{d+1}(0 \leq d \leq 7)$.

The behaviour at these boundaries was discussed in detail in [4,5], and is summarized as follows.

(i) The subgroup obtained by removing the root $\alpha_{d+1}$ associated with the last node $d+1$ of the Dynkin diagram. This is the "decompactification limit" in [4], in which the radius of one compact dimension, $r_{d} / \ell_{D+1}=$ $r^{2}$, becomes infinite, where $\ell_{D}$ is the $D$-dimensional Planck length. In this case the parabolic subgroup has a Levi factor, $M$, of the form $\mathrm{GL}(1) \times E_{d}$ and the constant term for any of the coefficient functions is a sum of a finite number of terms of the form $\sum_{i} r^{p_{i}} \mathcal{F}_{p_{i}}^{E_{d}}$ (suppressing some factors of $\log r$ ), where $\mathcal{F}_{p_{i}}^{E_{d}}$ is an automorphic function for the group $E_{d}$. This leads to a chain of relations from which it is possible to deduce all of the Eisenstein series from the $E_{8}$ case,

$$
E_{8} \supset E_{7} \supset E_{6} \supset \mathrm{SO}(5,5) \supset \mathrm{SL}(5) \supset \mathrm{SL}(3) \times \mathrm{SL}(2) \supset \mathrm{SL}(2)
$$

(ii) The subgroup obtained by removing the root $\alpha_{1}$ associated with the node 1. This is the "string perturbation theory limit" in [4], in which the amplitude is expanded for small string coupling, $y_{D}=r^{-4}$. In this case the parabolic subgroup has a Levi factor of the form GL(1) $\times$ $\mathrm{SO}(d, d)$ and the constant term is a sum of a finite number of terms of the form $\sum_{p} r^{p} \mathcal{F}_{p}^{\mathrm{SO}(d, d)}$. These correspond to terms in perturbative string theory, which have values that can be obtained by explicit integration over string world-sheets embedded in $\mathcal{M}^{10-d} \times \mathcal{T}^{d+1}$, where $\mathcal{M}^{D}$ is the $D$-dimensional Minkowski space.

(iii) The subgroup obtained by removing the root $\alpha_{2}$ associated to the node 2 . This is the limit in which the volume of the $M$-theory torus, $\mathcal{V}_{d+1} / \ell_{11}^{d+1}=r^{(2+2 d) / 3}$, becomes large. In this case the parabolic subgroup has a Levi factor of the form $\mathrm{GL}(1) \times \mathrm{SL}(d+1)$ and the constant term is a sum of a finite number of terms of the form $\sum_{p} r^{p} \mathcal{F}_{p}^{\mathrm{SL}(d+1)}$. In this limit the semi-classical approximation to 11-dimensional supergravity is a good approximation and the values of the constant terms 
can be determined by evaluating one and two-loop Feynman diagrams embedded in $\mathcal{M}^{10-d} \times \mathcal{T}^{d+1}$.

Detailed knowledge of these boundary conditions is nearly sufficient to determine the solutions to Equations (1.4) to (1.6). With these boundary conditions, we show that the solutions of (1.4) and (1.5) are sums of Eisenstein series defined with respect to specific parabolic subgroups of the group $E_{d+1}$ - up to the possible additive ambiguity of cusp forms. ${ }^{3}$ This was demonstrated in detail in [4] for $D \geq 6$, i.e., $d \leq 4$, and is generalized here to $d \leq 7$. Such cusp forms seem unlikely on purely mathematic grounds, because they have small Laplace eigenvalues. For example, the eigenvalues in (1.4) to (1.6) have the wrong sign to be part of the cuspidal spectrum unless $D$ is small enough. Even when the sign is correct, the papers $[19,20]$ give lower bounds on the cuspidal Laplace spectrum on $\operatorname{SL}(n, \mathbb{Z}) \backslash \operatorname{SL}(n, \mathbb{R}) /$ $\operatorname{SO}(n, \mathbb{R})$, for any $n$, which rule out such eigenvalues on this quotient. It seems plausible Langlands functorial lifting from the $E_{d+1} \operatorname{groups}$ to $\operatorname{SL}(n, \mathbb{R})$ could (at least conjecturally) reduce our cases of interest here to the results of $[19,20]$. Such a link would however require serious technical sophistication, and is beyond the scope of this paper.

In this paper we will extend this analysis to the remaining cases $5 \leq d \leq$ 7 , relating to $E_{6}, E_{7}$ and $E_{8}$. This involves a detailed analysis of constant terms of Eisenstein series for these groups, which will be the subject of section 2. The general analysis leads to very large numbers of power-behaved components in the constant terms. However, for the very special Eisenstein series of relevance to the string theory considerations there are immense simplifications and the relevant constant terms take the simple form expected according to items (i), (ii) and (iii). The application of these results into string theory language will be the subject of section 3 . There, it will be seen that there is precise agreement between the values of the constant terms and the expectations based on string theory.

\footnotetext{
${ }^{3}$ Cusp forms often arise as "error terms" in arithmetic expansions, dating back to the classical function $r_{4}(n)$, the number of ways an integer $n$ can be written as the sum of 4 squares. The generating function $\sum_{n>0} r_{4}(n) \mathrm{e}^{2 \pi \mathrm{i} n z}=\left(\sum_{n \in \mathbb{Z}} \mathrm{e}^{2 \pi \mathrm{i} n^{2} z}\right)^{4}$, and hence is a modular form of weight 2 for the congruence subgroup $\Gamma_{0}(4)$. There are no cusp forms of that weight for this group, and so the generating function is exactly an Eisenstein series - resulting in striking identities, such as $r_{4}(n)=8(n+1)$ for $n$ prime. However, as one looks at sums of more squares and the weight increases, cusp forms inevitably creep in and complicate the formulas. Our situation is similar: the existence of cusp forms would add a surprising touch of complexity to the Fourier coefficients and asymptotics of the solutions to (1.4) to (1.6).
} 
The complete expressions for the constant terms of relevance are contained in a number of tables in the Appendix.

The solutions of (1.6) are more general automorphic functions, $\mathcal{E}_{(0,1)}^{(10-d)}$. Their constant terms contain exponentially suppressed terms as well as terms that are powers of $r$ and were analysed for $d \leq 3$ in $[4,9,12]$, and for the case $d=4$ in $[5$, Appendix A]. The relevant constant terms for these coefficients in the $E_{8}$ case will be determined in section 4 . As we will show, the powerbehaved components of the constant term in the decompactification limit (i) for the $E_{8}$ case contain within them all three of the $E_{7}$ coefficients, $\mathcal{E}_{(0,0)}^{(4)}$, $\mathcal{E}_{(0,1)}^{(4)}$ and $\mathcal{E}_{(0,1)}^{(4)}$.

\section{Eisenstein series, parabolic subgroups and their constant terms}

This section contains an introduction to Langlands Eisenstein series on higher rank groups [21] and a description of some of his main results, followed by a computation of their constant terms in maximal parabolics. The discussion is geared towards the relevant setting of this paper, though we also make an effort to explain more general phenomena that may later be useful for string theorists. In particular, we mainly curtail the discussion to two particular types of Eisenstein series: minimal parabolic Eisenstein series, and maximal parabolic Eisenstein series induced from the constant function (which we shall see are specializations of the former).

We follow Langlands' Euler Products manuscript [22] in restricting to split Chevalley groups, as these are the only ones which arise in our investigations. In fact, we only need to study simply laced ones, i.e., either $G$ equals $\mathrm{SL}(n), \mathrm{SO}(n, n)$, or a split form of $E_{6}, E_{7}$ or $E_{8}$. Let $B$ denote a fixed minimal parabolic "Borel" subgroup of $G$. We decompose $B=M N$, where $M$ is its Levi component and $N$ its unipotent radical. The Cartan subgroup of $G$ shall be denoted by $A$.

\subsection{Eisenstein series in classical terminology}

Researchers in automorphic forms typically define Eisenstein series in terms of adele groups because of the computational benefits this framework affords. However, this is not necessary to state the definitions. In the present work it is important to understand the connection between Eisenstein series and other lattice constructions common in string theory. Hence we felt it appropriate to define the series in concrete terms, which we shall do in this subsection before recasting the definitions adelically in the next one. 
Let us now consider the real points $G(\mathbb{R})$ of $G$, and let $\Delta$ denote the roots of $G(\mathbb{R})$ relative to the Cartan $A(\mathbb{R})$. For each root $\alpha \in \Delta$, let $X_{\alpha}$ denote the Chevalley basis vector in the Lie algebra $\mathfrak{g}$ of $G(\mathbb{R})$ that represents it, and $n_{\alpha}(t)=\mathrm{e}^{t X_{\alpha}}$ the one-parameter unipotent subgroup it generates. Furthermore we may form the Cartan Lie element $H_{\alpha}=\left[X_{\alpha}, X_{-\alpha}\right] \in \mathfrak{a}$, the Lie algebra of $A(\mathbb{R})$. If $\Sigma^{+} \subset \Delta$ denotes the positive simple roots, then $\left\{H_{\alpha} \mid \alpha \in \Sigma^{+}\right\}$spans $\mathfrak{a}$. Thus we may identify elements of the connected component $A(\mathbb{R})^{0}$ of $A(\mathbb{R})$ with the exponentials $\mathrm{e}^{\sum_{\alpha \in \Sigma^{+}} c_{\alpha} H_{\alpha}}$, each $c_{\alpha}$ ranging over $\mathbb{R}$. The Iwasawa decomposition of $G(\mathbb{R})$ states that its elements $g$ each have unique decompositions $g=n a k$, with $n \in N(\mathbb{R}), a \in A(\mathbb{R})^{0}$ and $k \in K$, a maximal compact subgroup of $G(\mathbb{R})$. Thus there is a well-defined map $H: G(\mathbb{R}) \rightarrow \mathfrak{a}$ such that $g \in N \mathrm{e}^{H(g)} K$.

The roots $\alpha \in \Delta$ are by definition linear functionals on $\mathfrak{a}$, and every linear functional $\lambda \in \mathfrak{a}^{*} \otimes \mathbb{C}$ is a linear combination of elements of $\Sigma^{+}$with complex coefficients. In what follows it is helpful to normalize definitions using the linear functional $\rho$, defined to be half the sum of all positive roots. We denote the pairing between $\mathfrak{a}^{*} \otimes \mathbb{C}$ and $\mathfrak{a}$ by $\langle\cdot, \cdot\rangle$. When $H_{\alpha}$ and $\alpha$ are tacitly identified, this corresponds to the usual inner product for the root system. The Weyl group $\Omega$ acts both on $\mathfrak{a}$ and dually on $\mathfrak{a}^{*} \otimes \mathbb{C}$ in a way which preserves $\langle\cdot, \cdot\rangle$, and can be explicitly identified through any realization of the root system.

The function $H(g)$ is visibly unchanged if $g$ is multiplied on the left by an element of $N(\mathbb{R})$, and in particular any element of $N(\mathbb{Z})=N(\mathbb{R}) \cap G(\mathbb{Z})$, where $G(\mathbb{Z})$ is defined as in $[23]$, or equivalently as the stabilizer in $G(\mathbb{R})$ of the lattice spanned by the Chevalley basis [2]. It is likewise invariant under $A(\mathbb{Z})=A(\mathbb{R}) \cap G(\mathbb{Z})$ (because this finite group is contained in $K$ ), and hence under $B(\mathbb{Z})=B(\mathbb{R}) \cap G(\mathbb{Z})=N(\mathbb{Z}) A(\mathbb{Z})$ as well. Eisenstein series are formed by averaging such objects over cosets of a group modulo a subgroup it is invariant under:

Definition 2.1. The minimal parabolic Eisenstein series for $G$ is the coset sum

$$
E^{G}(\lambda, g):=\sum_{\gamma \in B(\mathbb{Z}) \backslash G(\mathbb{Z})} e^{\langle\lambda+\rho, H(\gamma g)\rangle} .
$$

This sum is absolutely convergent when the real part of $\lambda \in \mathfrak{a}^{*} \otimes \mathbb{C}$ has sufficiently large inner products with all $\alpha \in \Sigma^{+}$.

It is a famous result of Langlands that it meromorphically continues to all of $\mathfrak{a}^{*} \otimes \mathbb{C}$, to an automorphic function on $G(\mathbb{Z}) \backslash G(\mathbb{R})$. When $G=\operatorname{SL}(2, \mathbb{R})$ 
this definition recovers $\frac{1}{2 \zeta(2 s)} \sum_{(m, n) \in \mathbb{Z}^{2}-(0,0)}\left(\frac{y}{|m \tau+n|^{2}}\right)^{s}$, the usual non-holomorphic Eisenstein series for $\mathrm{SL}(2, \mathbb{Z})$. One can of course trivially modify the definition to apply to subgroups $\Gamma \subset G(\mathbb{Z})$, though this appears to be unnecessary for our investigations.

The power functions $e^{\langle\lambda+\rho, H(\gamma g)\rangle}$ and hence $E^{G}(\lambda, g)$ itself are always eigenfunctions of the Laplacian:

$$
\Delta^{G / K} E^{G}(\lambda, g)=2(\langle\lambda, \lambda\rangle-\langle\rho, \rho\rangle) E^{G}(\lambda, g) .
$$

This formula for the eigenvalue is crucial for identifying the solutions to (1.4) to (1.6), and is completely analogous to the $\mathrm{SL}(2)$ fact that $y^{s}$ is an eigenfunction of the hyperbolic Laplacian. It is proven by identifying $\Delta^{G / K}$ as a multiple of the Casimir on $G$, and using explicit formulas for the latter (see [24, p. 303]). Actually the power functions and hence Eisenstein series are eigenfunctions of not merely $\Delta^{G / K}$, but furthermore of the full ring of invariant differential operators. This is a crucial fact in Langlands' meromorphic continuation. So far string theoretic arguments have mainly produced information about $\Delta^{G / K}$ and not these other operators, so (2.3) naturally determines only $\langle\lambda, \lambda\rangle$. The structure of the constant terms and the integrality constraint discussed in section 3.1 is then used to pin down $\lambda$ exactly there.

We have used the term "minimal parabolic" series for these because of the role the Borel subgroup $B$ plays in their definition. In general, any subgroup $P$ that contains $B$ is called a standard parabolic subgroup; $P$ is called a maximal parabolic if $G$ itself is the only subgroup that properly contains it. All parabolic subgroups have the unique decomposition $P=$ $M_{P} N_{P}$, where $M_{P}$ is its Levi component and $N_{P}$ its unipotent radical. The standard parabolics of $G$ are in one-to-one correspondence with subsets $S \subset \Sigma^{+}$as follows: $M_{P}$ includes all $n_{ \pm \alpha}$ for $\alpha \notin S$, while $N_{P}$ contains all $n_{\alpha}$ for $\alpha \in S$. In particular, each maximal parabolic subgroup is associated to a single, simple root $\beta$, and we shall sometimes use the notation $P=P_{\beta}$ to emphasize this dependence.

Another family of important Eisenstein series that arise in our string theory calculations are maximal parabolic Eisenstein series. Let us first explain the simplest versions, which are induced from constant functions - they are in fact generalizations of the classical Epstein Zeta functions. These series are formed in a similar way to (2.2), but with special parameters $\lambda$ such that $\langle\lambda+\rho, H(g)\rangle$ is unchanged if $g$ is multiplied on the left by an element of $P(\mathbb{Z})$, where $P \supset B$ is a designated standard maximal parabolic subgroup of $G$. This is equivalent to requiring $\lambda+\rho$ be orthogonal to any simple root 
$\alpha$ other than the one $\beta$ which defines the maximal parabolic $P=P_{\beta}$, and restricts $\lambda$ to lie on a line in $\mathfrak{a}^{*} \otimes \mathbb{C}$. In terms of the dual basis $\left\{\omega_{\alpha} \mid \alpha \in \Sigma^{+}\right\}$ defined by the condition that $\left\langle\omega_{\alpha}, \beta\right\rangle=\delta_{\alpha=\beta}$, these $\lambda$ can be parametrized in terms of single complex variable $s$ as

$$
\lambda=2 s \omega_{\beta}-\rho
$$

The following definition uses this special choice of $\lambda$, but restricts the range of summation owing to the extra invariance of the summand under $P(\mathbb{Z})$ :

Definition 2.5. For $P=P_{\beta}$, the maximal parabolic Eisenstein series induced from the constant function is

$$
E_{\beta ; s}^{G}:=\sum_{\gamma \in P(\mathbb{Z}) \backslash G(\mathbb{Z})} \mathrm{e}^{2 s\left\langle\omega_{\beta}, H(\gamma g)\right\rangle} .
$$

Our normalization of $s$ is chosen so that it agrees with the usual nonholomorphic Eisenstein series for $\operatorname{SL}(2, \mathbb{Z})$. These series of course also have meromorphic continuations to $s \in \mathbb{C}$, and specialize to be identically equal to 1 at the special point $s=0$ because of the following fact (whose proof we shall describe later):

Theorem 2.7. The minimal parabolic Eisenstein series $E^{G}(-\rho, \cdot)$ equals the constant function 1.

To connect these two definitions, it is worthwhile to consider yet another type of maximal parabolic Eisenstein series that features another ingredient: an additional factor $\phi(\gamma g)$ in the summand (2.6), which is an automorphic function on the Levi component of $P$, extended to $G$. Such a sum is still well defined and has similar convergence properties. Definition 2.5 amounts to setting this function equal to 1 . Interestingly, the inclusion of this function allows us to view the maximal parabolic Eisenstein series (2.6) as a special case of (2.2). Indeed, recall the decomposition $P=M_{P} N_{P}$ from above, where $M_{P}$ is its Levi component and $N_{P}$ its unipotent radical. The intersection $B_{P}:=M_{P} \cap B$ is itself the Borel subgroup of the reductive group $M_{P}$, and the coset representatives $\gamma \in B(\mathbb{Z}) \backslash G(\mathbb{Z})$ can be uniquely decomposed into as products $\gamma=\gamma_{1} \gamma_{2}$ with $\gamma_{1} \in B_{P}(\mathbb{Z}) \backslash M_{P}(\mathbb{Z}), \gamma_{2} \in P(\mathbb{Z}) \backslash G(\mathbb{Z})$. Hence we may write $(2.2)$ as the double sum

$$
E^{G}(\lambda, g):=\sum_{\gamma_{1} \in B_{P}(\mathbb{Z}) \backslash M_{P}(\mathbb{Z})} \sum_{\gamma_{2} \in P(\mathbb{Z}) \backslash G(\mathbb{Z})} \mathrm{e}^{\left\langle\lambda+\rho, H\left(\gamma_{1} \gamma_{2} g\right)\right\rangle} .
$$


Any element $\lambda \in \mathfrak{a}^{*} \otimes \mathbb{C}$ can be uniquely decomposed as $\lambda=\lambda^{P}+\lambda_{P}$, where $\lambda^{P}$ is a complex linear combination of simple roots not equal to $\beta$ (the root defining $P=P_{\beta}$ ), and $\lambda_{P}$ is orthogonal to all such simple roots. This decomposition in particular applies to $\rho$, expressing it as the sum of $\rho^{P}$ (which is itself half the sum of the positive roots of $M_{P}$ ), and $\rho_{P}$ (which is a scalar multiple of $\omega_{\beta}$ ). Using this decomposition, we write the exponent as

$$
\begin{aligned}
\left\langle\lambda+\rho, H\left(\gamma_{1} \gamma_{2} g\right)\right\rangle & =\left\langle\lambda_{P}+\rho_{P}, H\left(\gamma_{1} \gamma_{2} g\right)\right\rangle+\left\langle\lambda^{P}+\rho^{P}, H\left(\gamma_{1} \gamma_{2} g\right)\right\rangle \\
& =\left\langle\lambda_{P}+\rho_{P}, H\left(\gamma_{2} g\right)\right\rangle+\left\langle\lambda^{P}+\rho^{P}, H\left(\gamma_{1} \gamma_{2} g\right)\right\rangle ;
\end{aligned}
$$

in the last step we have used the fact that $H(m g)$ and $H(g)$ have the same inner product with $\lambda_{P}+\rho_{P}$, for any $m$ generated by the $n_{ \pm \alpha}$ for $\alpha \in \Sigma_{\neq \beta}^{+}$, in particular $M_{P}(\mathbb{Z})$. Hence $(2.8)$ can be expressed in the range of absolute convergence as

$$
E^{G}(\lambda, g):=\sum_{\gamma_{2} \in P(\mathbb{Z}) \backslash G(\mathbb{Z})} \mathrm{e}^{\left\langle\lambda_{P}+\rho_{P}, H\left(\gamma_{2} g\right)\right\rangle} \phi\left(\gamma_{2} g\right),
$$

where $\phi(g):=\sum_{\gamma_{1} \in B_{P}(\mathbb{Z}) \backslash M_{P}(\mathbb{Z})} \mathrm{e}^{\left\langle\lambda^{P}+\rho^{P}, H\left(\gamma_{1} g\right)\right\rangle}$ is now a minimal parabolic Eisenstein series for the smaller reductive group $M_{P}$. Thus minimal parabolic Eisenstein series are themselves special cases of maximal parabolic Eisenstein series - but induced from the function $\phi$ rather than the constant function.

We can now see that (2.6) is a specialization that coincides with (2.2) when $\lambda$ has the form (2.4). In this case $\lambda^{P}=-\rho^{P}$, and the Eisenstein series on $M_{P}$ in the previous paragraph specializes to be constant because of Theorem 2.7. Hence under the special assumption (2.4), the inducing function $\phi$ is constant and the two notions coincide.

\subsection{Eisenstein series in adelic terminology}

We have just given definitions of the Eisenstein series involved in this paper, in concrete classical terms. Adele groups are often used in automorphic forms as a notational simplification that hints to effective ways to group terms together in calculations. In the context of Eisenstein series, they are used to reparametrize the sums over $P(\mathbb{Z}) \backslash G(\mathbb{Z})$ (whose cosets can be intricate to describe). This application — a brilliant insight of Piatetski-Shapiro that was furthered by Langlands - has been crucial in readily obtaining exact formulas (by comparison, Poisson summation is much harder to execute directly). Since this is crucial to our calculations, we have elected to give a description here. 
Let us return to $(2.2)$ and its sum over cosets $B(\mathbb{Z}) \backslash G(\mathbb{Z})$. As we mentioned above, cosets for this quotient can be difficult to directly describe, especially as the group $G$ gets more complicated. This is because $\mathbb{Z}$ is a ring, not a field like $\mathbb{R}$ (where the corresponding quotient is just the maximal compact subgroup $K)$. Strikingly, the field $\mathbb{Q}$ gives the same coset space as $\mathbb{Z}$ : the inclusion map from $G(\mathbb{Z})$ into $G(\mathbb{Q})$ induces the bijection of cosets

$$
B(\mathbb{Z}) \backslash G(\mathbb{Z}) \simeq B(\mathbb{Q}) \backslash G(\mathbb{Q}) .
$$

This is because our assumptions on $G$ imply that $G(\mathbb{Q})=B(\mathbb{Q}) G(\mathbb{Z})$ (see $[22$, $\S 2]$ ). Similarly, $P(\mathbb{Z}) \backslash G(\mathbb{Z}) \simeq P(\mathbb{Q}) \backslash G(\mathbb{Q})$, and therefore our Eisenstein series can, in principle, be written as sums over these rational quotients. To do this properly one needs to redefine $H$ in such as away that it is invariant under $B(\mathbb{Q})$ on the left. Note that the existing definition does not qualify, because $A(\mathbb{Q})$ is dense in $A(\mathbb{R})$ and hence $H$ cannot be trivial on it.

The remedy is to instead consider each of the groups $G\left(\mathbb{Q}_{p}\right)$, where $p$ denotes either a prime number or $\infty$ (in the latter situation, we follow the convention that $\left.\mathbb{Q}_{\infty}=\mathbb{R}\right)$. Just as the adeles $\mathbb{A}$ are the restricted product of all $\mathbb{Q}_{p}$ with respect to the $\mathbb{Z}_{p}$ (i.e., all but a finite number of components of each element lie in $\mathbb{Z}_{p}$ ), the adele group $G(\mathbb{A})$ is the restricted product of each $G\left(\mathbb{Q}_{p}\right)$ with respect to the $G\left(\mathbb{Z}_{p}\right)=$ the stabilizer of the Chevalley lattice, tensored with $\mathbb{Z}_{p}$.

A variant of the Iwasawa decomposition persists in the $p$-adic case as well: $G\left(\mathbb{Q}_{p}\right)=N\left(\mathbb{Q}_{p}\right) A\left(\mathbb{Q}_{p}\right) G\left(\mathbb{Z}_{p}\right)$, though this is no longer unique since, for example, $A\left(\mathbb{Q}_{p}\right) \cap G\left(\mathbb{Z}_{p}\right)$ is nontrivial. Thus, globally, we have that $G(\mathbb{A})=$ $N(\mathbb{A}) A(\mathbb{A}) K_{\mathbb{A}}$, where $K_{\mathbb{A}}$ is the product of the real group $K$ with all $G\left(\mathbb{Z}_{p}\right)$ 's. Since $G$ is assumed to be split, $A(\mathbb{A})$ is a product of $r=\operatorname{rank}(G)$ copies of the torus $\mathrm{GL}(1, \mathbb{A})=\mathbb{A}^{*}$, the ideles of $\mathbb{A}$. Strong approximation for the ideles equates the quotient $\mathbb{Q}^{*} \backslash \mathbb{A}^{*}$ with $\left(\{ \pm 1\} \backslash \mathbb{R}^{*}\right) \times \widehat{\mathbb{Z}}^{*}$, where $\mathbb{Q}$ is regarded as diagonally ${ }^{4}$ embedded into $\mathbb{A}$, and $\widehat{\mathbb{Z}}^{*}$ is the product of all $\mathbb{Z}_{p}^{*}$ s. Since the first factor is isomorphic to $\mathbb{R}$ via the logarithm map, this identification extends $H$ from a function on $A(\mathbb{R}) \simeq\left(\mathbb{R}^{*}\right)^{r}$ to an $A(\mathbb{Q})$-invariant, $A\left(\widehat{\mathbb{Z}}^{*}\right)$ invariant function from $A(\mathbb{A})$ to $\mathfrak{a}$. Furthermore, it extends to $G(\mathbb{A})$ through the global Iwasawa decomposition to a left $B(\mathbb{Q})$-invariant function, with $B(\mathbb{Q})$ likewise thought of as being diagonally embedded into $G(\mathbb{A})$. We can now define the adelic Eisenstein series as

$$
E^{G}(\lambda, g)=\sum_{\gamma \in B(\mathbb{Q}) \backslash G(\mathbb{Q})} \mathrm{e}^{\langle\lambda+\rho, H(\gamma g)\rangle}
$$

\footnotetext{
${ }^{4}$ i.e., into each factor $\mathbb{Q}_{p}$ simultaneously.
} 
which we stress agrees with (2.2) when the argument $g \in G(\mathbb{R})$, and defines an extension to $g \in G(\mathbb{A})$ that is left-invariant under the diagonally embed$\operatorname{ded} G(\mathbb{Q})$. Similarly

$$
E_{\beta ; s}^{G}:=\sum_{\gamma \in P(\mathbb{Q}) \backslash G(\mathbb{Q})} \mathrm{e}^{2 s\left\langle\omega_{\beta}, H(\gamma g)\right\rangle},
$$

which is again a specialization of (2.12) under the assumption (2.4).

\subsection{Constant term formulas}

In general the Fourier expansions of Eisenstein series are intricate to state, and are not presently known in full detail. However, a simple natural part of them (defined as follows) have very explicit formulas due to Langlands, and are crucial to the analytic properties of these series.

Definition 2.14. The constant term of an automorphic form $\phi$ in a standard parabolic subgroup $P \supset B$ is given by the integral

$$
\int_{N_{P}(\mathbb{Z}) \backslash N_{P}(\mathbb{R})} \phi(n g) d n
$$

where $N_{P}$ is the unipotent radical of $P$, and $d n$ is Haar measure on $N_{P}$ normalized to give the quotient $N_{P}(\mathbb{Z}) \backslash N_{P}(\mathbb{R})$ volume 1 . (Since $N_{P}$ is unimodular, dn is simultaneously both a left and right Haar measure.)

We now state the constant term formula for the minimal parabolic Eisenstein series $E^{G}(\lambda, g)$ in maximal parabolics. When (2.4) holds, the specialization of the formula below gives the constant terms of the maximal parabolic series in Definition 2.5 - this will be very useful for our applications. The formula involves the functions

$$
M(w, \lambda)=\prod_{\substack{\alpha \in \Delta^{+} \\ w \alpha \in \Delta^{-}}} c(\langle\lambda, \alpha\rangle), \quad c(s)=c(-s)^{-1}=\frac{\xi(s)}{\xi(s+1)}
$$

which arise from intertwining operators. Here $w$ is an element of the Weyl group $\Omega$ of $G, \lambda \in \mathfrak{a}^{*} \otimes \mathbb{C}$, and $\xi(s)$ is the completed Riemann $\zeta$-function 
$\pi^{-s / 2} \Gamma\left(\frac{s}{2}\right) \zeta(s)$. They satisfy the cocycle identity

$$
M\left(w_{1} w_{2}, \lambda\right)=M\left(w_{1}, w_{2} \lambda\right) M\left(w_{2}, \lambda\right)
$$

Let $P=P_{\gamma}$ be any maximal parabolic, and let $\Omega_{P}$ denote the Weyl group of $M_{P}$, thought of as a subgroup of $\Omega$. As above, we decompose any $\lambda \in \mathfrak{a}^{*} \otimes \mathbb{C}$ as the sum $\lambda_{P}+\lambda^{P}$, where $\lambda_{P}$ is perpendicular to all simple roots aside from $\gamma$, and $\lambda^{P}$ is a multiple of $\omega_{\gamma}$.

Theorem 2.18. (Langlands' Constant term formula - see [25, Proposition II.1.7.ii, p. 92])

$$
\begin{aligned}
& \int_{N_{P}(\mathbb{Z}) \backslash N_{P}(\mathbb{R})} E^{G}(\lambda, n g) d n \\
& \quad=\sum_{w \in \Omega_{P} \backslash \Omega} \mathrm{e}^{\left\langle(w \lambda)_{P}+\rho_{P}, H(g)\right\rangle} M(w, \lambda) E^{M_{P}}\left((w \lambda)^{P}, g\right) .
\end{aligned}
$$

Of course the last Eisenstein series $E^{M_{P}}$ must be thought of as a product of Eisenstein series on the different reductive factors of $M_{P}$ when the latter is not simple. Moeglin-Waldspurger actually prove a slightly different statement, where the $M(w, \lambda)$ appear directly as intertwining operators which satisfy a composition law identically compatible to (2.17). Using embedded SL(2)'s inside $G$ it is easy to see that these operators act there as the scalar (2.16) when $w$ is a simple Weyl reflection, and therefore on the full Weyl group as well. Moeglin-Waldspurger also deal with adelic Eisenstein series, which here can be equated to their classical variants via the correspondence described in the previous subsection. Also, the adelic constant term integration over $N_{P}(\mathbb{Q}) \backslash N_{P}(\mathbb{A})$ there drops to $N_{P}(\mathbb{Z}) \backslash N_{P}(\mathbb{R})$ for the adelization of classical Eisenstein series (this is because of strong approximation for $\mathbb{A})$. Strictly speaking, we have also used the fact that the Weyl group of a Chevalley group sits inside $G(\mathbb{Z})$.

Formula (2.19) is consistent with two other important identities about the minimal parabolic Eisenstein series: the functional equation

$$
E^{G}(\lambda, g)=M(w, \lambda) E^{G}(w \lambda, g), \quad w \in \Omega
$$

and Langlands' constant terms formula in the minimal parabolic

$$
\int_{N(\mathbb{Z}) \backslash N(\mathbb{R})} E^{G}(\lambda, n g) d n=\sum_{w \in \Omega} \mathrm{e}^{\langle w \lambda+\rho, H(g)\rangle} M(w, \lambda) .
$$


For example, when $\lambda=-\rho$ the inner product of $\lambda$ and any simple root is -1 , a point at which $c(s)$ vanishes. Any nontrivial Weyl group element flips the sign of at least one simple root, so that $M(w, \lambda)$ vanishes for all but the identity element $w \in \Omega$. This means the constant term (2.21) is identically one, consistent with Theorem 2.7. Moreover, it is not hard to deduce Theorem 2.7 from this calculation. Indeed, the general theory of Eisenstein series describes how the constant terms control the growth of Eisenstein series: since this one is bounded, so is the full series. It is furthermore a Laplace eigenfunction with eigenvalue 0 when $\lambda=-\rho$, and hence it is constant.

As noted above, because $E^{G}$ specializes to the maximal parabolic Eisenstein series under (2.4), Theorem 2.18 provides the constant terms of those objects as well. It is possible to prove those formulas more directly, without reference to the minimal parabolic Eisenstein series, for example as is argued for some of the relevant cases earlier in [26]. However, we felt it was important to calculate everything via the minimal parabolic Eisenstein series for two different reasons. The first is that we will rely heavily on special identities relating various $E_{\beta ; s}^{G}$ via $(2.20)$ that are more apparent as specializations, rather than as identities of sums over completely different coset spaces. The second reason is that the constant term calculation in the proof of [26, Theorem 2.3] does not carry over to all Eisenstein series, because they assert that the intersection of the Levi component of a maximal parabolic with the conjugate of another parabolic is itself a maximal parabolic subgroup of this Levi. However, this is false in general - including for a number of subtle examples we faced in the present work, such as the case of $G=E_{6}$ when $\beta=\alpha_{5}$ and the constant term is taken in the maximal parabolic corresponding to $\alpha_{1}$ (in the numbering of figure 1). We would like to make clear that this in no way affects the validity of the results in [26], because the assertion is valid in the cases they study — rather it only affects extensions of their work to different situations. What it means is that non-maximal parabolic Eisenstein series can arise in some of the constant terms we study. Strikingly, these series tend to vanish at the special points of interest.

Finally, in addition to terms vanishing because a simple root is flipped, sometimes Eisenstein series vanish for a more subtle reason: all their constant terms vanish. In such a case, the Eisenstein series is by definition a cusp form, which much itself vanish because Eisenstein series are orthogonal to all cusp forms. It follows by induction that this is the case if and only if (2.21) vanishes, which can happen even at points where the individual $M(w, \lambda)$ are singular because of cancellation between terms. This can be very tedious to computationally check, even with the methods of the paragraph below. This 
vanishing, however, is ultimately responsible for many of our simple formulas for constant terms at special points.

\subsection{Brief description of explicit computations used later in the paper}

Theorem 2.18 gives explicit formulas for all constant terms we are after, since it is possible to enumerate the Weyl group, describe its action on $\lambda$ and calculate the exact factors $M(w, \lambda)$ from (2.16). In practice, the Weyl group is so large that it is difficult to have an a priori explanation of what the calculation will give. Langlands noticed already in his example of the rank two group $G_{2}$ in [21, Appendix 3] that many terms vanish at special points. Indeed, such vanishing is absolutely crucial to the physical conclusions we draw from the case of $E_{8}$ and its subgroups. Mathematicians have studied similar vanishing in different settings, and giving explanations for it (e.g., $[26,27])$.

Unfortunately, it was not initially obvious what configurations of parabolics $P_{\gamma}$ and maximal parabolic Eisenstein series $E_{\beta ; s}^{G}$ to investigate, and it became a practical necessity to have a fast way to obtain explicit constant terms for large swaths of examples in order to unravel structures which were important to us from a string-theoretic point of view. Economical mathematical explanations for the phenomena at hand were not as important as speed, especially in light of the complexity of the objects involved. To get around this issue, we first precomputed which Weyl group elements $w$ flip a simple root $\alpha \neq \beta$. For such $\alpha,\left\langle 2 s \omega_{\beta}-\rho, \alpha\right\rangle=-1$, which forces the product (2.16) to vanish. These terms, which provide the vast majority, can hence be discarded from the constant term calculations. The remaining terms are stored for later calculations. For example, out of the $696,729,600$ $E_{8}$ Weyl group elements, only 240 are needed when $\beta$ is the 8 -th root of $E_{8}$, i.e., the one which is added on from $E_{7}$. To lessen storage requirements, we worked with a factorization of the $E_{n}$ Weyl groups in terms of the $E_{n-1}$ Weyl group, and a fixed set of coset representatives. The resulting computer calculations were then very efficient, and allowed us to explore the properties of a large number of cases and identify significant patterns. Ultimately the constant term calculations were automated, only rarely taking more than 30 seconds a piece.

The constant term is a function on the Levi component $M_{P}$ of the maximal parabolic $P=P_{\gamma}$, which is the product of a one-dimensional group with all Chevalley groups whose Dynkin diagrams compose the connected components of the Dynkin diagram of $G$, once the node for the simple root 
$\gamma$ is deleted (note that this is not in general the same root $\beta$ that defines the Eisenstein series). This second factor is the group that the Eisenstein series $E^{M_{P}}$ on the right-hand side of (2.19) is defined on. The exponential factor $\mathrm{e}^{\left\langle(w \lambda)_{P}+\rho_{P}, H(g)\right\rangle}$ multiplying it depends on the one-dimensional piece. To parameterize it uniformly, factor $g \in M_{P}(\mathbb{R})$ as $t g_{0}$, where $t$ is in this one-dimensional piece and $g_{0}$ is in the product of smaller Chevalley groups. By construction, $t$ acts trivially on the roots spaces spanned by the $X_{\alpha}$ contained inside $M_{P}$, and is uniquely determined by its eigenvalue on $X_{\gamma}$, which we parametrize as $2 \log r$. Hence the exponential factor in (2.19) is a power of $r$, and the constant term is a polynomial with exponents depending on $s$ whose coefficients are lower-rank Eisenstein series.

Example. $G=E_{8}, \beta=\alpha_{1}, \gamma=\alpha_{2}$.

Here all but 2160 out of the 696,729,600 Weyl group elements give zero contribution, and the polynomial just mentioned is equal to $420 r^{84}+8 r^{230-20 s}+168 r^{162-12 s}+280 r^{134-8 s}+8 r^{140-8 s}+56 r^{114-4 s}+$ $280 r^{-4(-27+s)}+70 r^{-16(-12+s)}+8 r^{20 s}+168 r^{12(2+s)}+8 r^{8(6+s)}+56 r^{4(17+s)}+$ $280 r^{62+4 s}+280 r^{42+8 s}+70 r^{8+16 s}$, provided the Eisenstein series are suppressed (otherwise the formula is even more unwieldy). At the special point $s=\frac{3}{2}$ all but the two smallest powers of $r$ vanish, giving

$$
r^{30} E_{\alpha_{1} ; \frac{3}{2}}^{A_{7}}+\frac{\xi(2)}{\xi(3)} r^{32},
$$

as the constant term.

\section{String theory amplitudes and their degeneration limits}

The results of the previous section allow us to extend the analysis of the structure of the automorphic functions that arise in the expansion of the string theory amplitude [4] to a more general setting that includes the exceptional duality groups.

It is useful to translate the terms in the low-energy expansion of the analytic part of the scattering amplitude, (1.3) into local terms in an effective action, so that the first three terms beyond classical Einstein theory in $D$ dimensions are

$$
\begin{array}{r}
S_{R^{4}}=\ell_{D}^{8-D} \int d^{D} x \sqrt{-G^{(D)}} \mathcal{E}_{(0,0)}^{(D)} \mathcal{R}^{4}, \\
S_{\partial^{4} R^{4}}=\ell_{D}^{12-D} \int d^{D} x \sqrt{-G^{(D)}} \mathcal{E}_{(1,0)}^{(D)} \partial^{4} \mathcal{R}^{4},
\end{array}
$$


and

$$
S_{\partial^{6} R^{4}}=\ell_{D}^{14-D} \int d^{D} x \sqrt{-G^{(D)}} \mathcal{E}_{(0,1)}^{(D)} \partial^{6} \mathcal{R}^{4}
$$

We will first consider the solutions for the coefficients $\mathcal{E}_{(0,0)}^{(D)}$ and $\mathcal{E}_{(1,0)}^{(D)}$, which satisfy the Laplace eigenvalue equations (1.4) and (1.5). The discussion of the automorphic coefficient $\mathcal{E}_{(0,1)}^{(D)}$, which satisfies the more elaborate equation (1.6), will be deferred to section 4 .

Note on conventions. The solutions will involve linear combinations of Eisenstein series of the kind described in the last section. In describing the string theory results it will prove convenient to use a normalization for maximal parabolic Eisentein series that includes a factor of $2 \zeta(2 s)$, so we will define

$$
\mathbf{E}_{\left[0^{u} 10^{d-u}\right] ; s}^{E_{d+1}}:=2 \zeta(2 s) E_{\beta ; s}^{E_{d+1}}
$$

where $\left[0^{u} 10^{d-u}\right]$ is the Dynkin label associated with the simple root $\beta$ in the definition (2.4). Furthermore, since the conventional SL(2) Eisenstein series has a trivial Dynkin label it will be written as $\mathbf{E}_{s}^{\mathrm{SL}(2)}$.

The parameter $r$, defined in section 2.4, associated with the GL(1) factor in section 2 translates into distinct physical parameters in each of the three degeneration limits that correspond to deleting nodes $d+1,1$ and 2 , respectively, of the Dynkin diagram in figure 1 . These are summarized as follows:

Limit (i) $\quad r^{2}=r_{d} / \ell_{D+1}, \quad\left(r_{d}=\right.$ radius of circle $)$,

Limit (ii) $\quad r^{-4}=y_{D}=$ string term constant,

Limit (iii) $\quad r^{\frac{2+2 d}{3}}=\mathcal{V}_{d+1} / \ell_{11}^{d+1}, \quad \mathcal{V}_{d+1}=$ volume of $\mathrm{M}$ theory torus.

The $D$-dimensional string coupling constant is defined by $y_{D}=g_{s}^{2} \ell_{s}^{d} / V_{d}$, where $g_{s}$ is the $D=10$ IIA or IIB string coupling and $V_{d}$ is the volume of $\mathcal{T}^{d}$ in string units. The Planck scales in different dimensions that enter in (3.1) and (3.2) are related to each other and the string scale, $\ell_{s}$, by

$$
\ell_{D}^{D-2}=\ell_{D+1}^{D-1} \frac{1}{r_{d}}=\ell_{s}^{D-2} y_{D} \quad \text { for } D \leq 10, \quad \ell_{11}=g_{A}^{\frac{1}{3}} \ell_{s}
$$

where $g_{A}$ is the IIA string coupling. 


\subsection{Solutions for the coefficients $\mathcal{E}_{(0,0)}^{(D)}$ and $\mathcal{E}_{(1,0)}^{(D)}$}

We will show that the automorphic coefficients in (3.1) and (3.2) are given by the simple expressions:

$$
\mathcal{E}_{(0,0)}^{(D)}=2 \zeta(3) E_{\alpha_{1} ; \frac{3}{2}}^{G}:=\mathbf{E}_{\left[10^{d}\right] ; \frac{3}{2}}^{E_{d+1}}
$$

and

$$
\mathcal{E}_{(1,0)}^{(D)}=\zeta(5) E_{\alpha_{1} ; \frac{5}{2}}^{G}:=\frac{1}{2} \mathbf{E}_{\left[10^{d}\right] ; \frac{5}{2}}^{E_{d+1}},
$$

for $3 \leq D \leq 5$ (or $7 \geq d \geq 5$ ). Substituting the expression (2.4) for $\lambda$ in terms of $s$ into Equation (2.3), it follows that the solutions (3.6) and (3.7) satisfy the Laplace eigenvalue Equations (1.4) and (1.5) with $s=3 / 2$ and $s=5 / 2$, respectively. We will shortly show that these functions also satisfy the requisite boundary conditions in the three limits of interest. An important general comment about these boundary conditions is that in each of the three limits the automorphic coefficients have moderate power-like growth for $r \rightarrow \infty$. Another necessary condition for these being acceptable solutions is that in the limits (i) and limit (ii) they give rise to integer powers of the radius $r_{d} / \ell_{D+1}=r^{2}$ and the string coupling $y_{D}=r^{-4}$. Strictly speaking our calculations merely show that (3.6) and (3.7) solve all relevant equations, but do not directly rigorously show that they are unique solutions. However, any two solutions differ only by a linear combination of cusp forms and other Eisenstein series. The constant terms of these series can be computed explicitly as well. Though we do not fully investigate this here, the possibility of other solutions seems unlikely because of known non-existence results for cusp forms on $\mathrm{SL}(n, \mathbb{Z}) \backslash \mathrm{SL}(n, \mathbb{R}) / \mathrm{SO}(n, \mathbb{R})[19,20]$, the conjectured properties of Langlands' functorial lifts and the rationality of the cuspidal eigenvalues the above integrality constraint dictates.

First we will comment on the form of these solutions. From the general expression (2.4) for the weight vector that defines a maximal parabolic Eisenstein series, the vector associated with a maximal parabolic Eisenstein series $E_{\beta ; s}^{G}$ is $\lambda_{\beta}(s)=2 s \omega_{\beta}-\rho$. For the series in (3.6) and (3.7) this has the form $\lambda_{\alpha_{1}}(s)=2 s \omega_{\alpha_{1}}-\rho$, where $\omega_{\alpha_{1}}$ is the weight vector for the simple root $\alpha_{1}$ labelling the first node of the Dynkin diagram in figure 1. Therefore, the weight vector associated with the series $E_{\alpha_{1} ; 3 / 2}^{E_{d+1}}$ is $\lambda_{\alpha_{1}}(3 / 2)$, while for the series $E_{\alpha_{d+1} ; 5 / 2}^{E_{d+1}}$ it is $\lambda_{\alpha_{1}}(5 / 2)$. As we know from earlier examples, there are many equivalent ways of expressing the same series as those in (3.6) 
and (3.7). For the exceptional groups, $E_{d+1}$ with $d=5,6,7$, the weight vectors $\lambda_{\alpha_{1}}(3 / 2), \lambda_{\alpha_{2}}(1)$ and $\lambda_{\alpha_{d+1}}((d-2) / 2)$ are in the same orbit under the action of the Weyl group, $\Omega$. Similarly, $\lambda_{\alpha_{1}}(5 / 2)$ and $\lambda_{\alpha_{d+1}}((d+2) / 2)$ are also in the same Weyl orbit. This means that as a consequence of the functional equation (2.20) satisfied by the minimal parabolic Eisenstein series, the maximal parabolic Eisenstein series satisfy the following relationships, among many others:

$$
\begin{aligned}
& \mathbf{E}_{\left[10^{d}\right] ; \frac{3}{2}}^{E_{d+1}} \propto \mathbf{E}_{\left[010^{d-1}\right] ; 1}^{E_{d+1}} \propto \mathbf{E}_{\left[0^{d} 1\right] ; \frac{d-2}{2}}^{E_{d+1}}, \\
& \mathbf{E}_{\left[10^{d}\right] ; \frac{5}{2}}^{E_{d+1}} \propto \mathbf{E}_{\left[0^{d} 1\right] ; \frac{d+2}{2}}^{E_{d+1}} .
\end{aligned}
$$

The symbol $\propto$ means that the quantities are equal up to a constant of proportionality, which may depend on $d$. In this manner our solutions can be rewritten in many different ways. Such a relationship was pointed out in the $s=3 / 2$ case in [13].

We will now check that the solutions in (3.6) and (3.7) behave in the appropriate manner in the three degeneration limits described in the Introduction.

\section{(i) Decompactification from $D$ to $D+1$}

This is the limit associated with the parabolic subgroup $P_{\alpha_{d+1}}$, for node $d=10-D$. Consistency under decompactification in this limit $r_{d} / \ell_{D+1} \gg 1$ requires (see [5, Equations (2.13) and (2.15)]),

$$
\int_{P_{\alpha_{d+1}}} \mathcal{E}_{(0,0)}^{(D)} \simeq \frac{\ell_{D+1}^{8-D}}{\ell_{D}^{8-D}}\left(\frac{r_{d}}{\ell_{D+1}} \mathcal{E}_{(0,0)}^{(D+1)}+\left(\frac{r_{d}}{\ell_{D+1}}\right)^{8-D}\right)
$$

and

$$
\begin{aligned}
\int_{P_{\alpha_{d+1}}} \mathcal{E}_{(1,0)}^{(D)} \simeq & \frac{\ell_{D+1}^{12-D}}{\ell_{D}^{12-D}}\left(\frac{r_{d}}{\ell_{D+1}} \mathcal{E}_{(1,0)}^{(D+1)}\right. \\
& \left.+\left(\frac{r_{d}}{\ell_{D+1}}\right)^{6-D} \mathcal{E}_{(0,0)}^{(D+1)}+\left(\frac{r_{d}}{\ell_{D+1}}\right)^{12-D}\right)
\end{aligned}
$$

The symbol $\simeq$ means that constant factors multiplying each term have been suppressed.The fact that, for $D=3,4,5$ (i.e., for the duality groups $E_{6}$, $E_{7}$ and $E_{8}$ ) the automorphic coefficients in these expressions are simply given by the Eisenstein series shown in (3.6), (3.7) follows from the precise 
expressions given in the first three rows of tables A.4 and A.6 (where the parameter $r$ used in these expressions is given by $\left.r^{2}=r_{d} / \ell_{D+1}\right)$.

The terms linear in $r_{d}$ in (3.9) and (3.10) are the ones that contain the coefficients of the interactions in $D+1$ dimensions in the large- $r_{d}$ limit, as can be seen by substituting into (3.1) and (3.2). The other terms contribute in a well-understood way to the nonanalytic part of the amplitude, $A^{\text {nonan }}$ in (1.1) (or, equivalently, to nonlocal terms in the effective action) [4]. Schematically, the last terms in parentheses are the $n=0$ and $n=2$ terms in an infinite series of the form $r_{d}^{8-D} \sum_{n=0}^{\infty} c_{n}\left(r_{d}^{2} s\right)^{n} \mathcal{R}^{4}$ that gives the supergravity threshold behaviour $s^{(D-8) / 2} \log s \mathcal{R}^{4}$ (D even) or $s^{(D-8) / 2} \mathcal{R}^{4}$ ( $D$ odd), in the limit $r_{d}^{2} s \rightarrow \infty$ (this is the standard one-loop supergravity threshold). This can be described as the effect of the sum over an infinite set of thresholds for Kaluza-Klein modes of mass $p / r_{d}$ (positive integer $p$ ) in the limit that they become massless. The second term in parentheses in (3.10) is similarly the $n=0$ contribution to a second infinite series of powers, $r_{d}^{2-D} \sum_{n=0}^{\infty} c_{n}^{\prime}\left(r_{d}^{2} s\right)^{n+2}$, which sum up to give a threshold that arises at order $\ell_{D}^{6}$. The structure of this term is determined by unitarity in the manner described in [28, Section 3].

Using the relationships derived in section 2 it is straightforward to check that these properties are satisfied by expressions (3.6) and (3.7). In this manner it is clear that the chain of coefficients for dimensions $3 \leq D \leq 10$ can be obtained from the functions for $D=3$ (the $E_{8}$ case) in (3.6) and (3.7). Furthermore, expression (3.10) shows that both the coefficients $\mathcal{E}_{(0,0)}^{(D+1)}$ and $\mathcal{E}_{(1,0)}^{(D+1)}$ are contained in the constant term for $\mathcal{E}_{(0,0)}^{(D)}$ in the parabolic subgroup $P_{\alpha_{d+1}}$. In the next section we will see that the coefficient $\mathcal{E}_{(0,1)}^{(D)}$ contains all three functions $\mathcal{E}_{(0,0)}^{(D+1)}, \mathcal{E}_{(1,0)}^{(D+1)}$ and $\mathcal{E}_{(0,1)}^{(D+1)}$ in its constant term in the $P_{\alpha_{d+1}}$ parabolic. In particular, we will show that starting from the largest-rank case, $D=3$ with the group $E_{8}$, the complete towers of all three coefficients can be obtained by starting from the single coefficient, $\mathcal{E}_{(1,0)}^{(3)}$.

Intriguingly, line three of table A.6 shows that the coefficient $\mathcal{E}_{(1,0)}^{(D)}$ demonstrates a bifurcation under the decompactification from $D=5$ to $D=6$, so that for $D=6$ this coefficient is the sum of two Eisenstein series (as suggested in [4]). This point merits special discussion, which will be given in subsection 3.2 .

\section{(ii) The perturbative limit}

This limit is associated with the parabolic subgroup $P_{\alpha_{1}}$ and is given by $y_{D} \rightarrow 0$ with $\ell_{s}$ fixed. In this limit the expansions of the interactions (3.1) 
and (3.2) are given by the constant terms [4]

$$
\ell_{D}^{8-D} \int_{P_{\alpha_{1}}} \mathcal{E}_{(0,0)}^{(D)} \simeq \ell_{s}^{8-D}\left(\frac{2 \zeta(3)}{y_{D}}+\mathbf{E}_{\left[10^{d-1}\right] ; \frac{d}{2}-1}^{\mathrm{SO}(d, d)}\right)
$$

and

$$
\ell_{D}^{12-D} \int_{P_{\alpha_{1}}} \mathcal{E}_{(1,0)}^{(D)} \simeq \ell_{s}^{12-D}\left(\frac{\zeta(5)}{y_{D}}+\mathbf{E}_{\left[10^{d-1}\right] ; \frac{d}{2}+1}^{\mathrm{SO}(d, d)}+y_{D} \mathbf{E}_{\left[0^{d-2} 10\right] ; 2}^{\mathrm{SO}(d, d)}\right)
$$

Once again, the precise numerical coefficients are given in tables A.4 and A.7 with the relation $r^{4}=1 / y_{D}$.

A term of order $y_{D}^{-1+h}$ should be interpreted as a genus- $h$ perturbative string theory contribution to the amplitude that can be calculated by functional integration of a genus- $h$ world-sheet embedded in toroidally compactified Minkowski space, which reduces to an integral over the moduli of the world-sheet. This can be checked explicitly by extending the methods of $[29,30]$ to compactification on $\mathcal{T}^{d}$ with $d>2$ and to $h \geq 1$. This leads to expressions for terms in the expansion of the genus- $h$ amplitude of the form $\sigma_{2}^{p} \sigma_{3}^{q} \mathcal{R}^{4} I_{h}^{(d)}\left[j_{h}^{(p, q)}\right]$, where $j_{h}^{(p, q)}$ are functions of the world-sheet moduli that are, in principle, determined by expanding the genus- $h$ amplitude, although in practise this has only been done in detail for $h=1$ in $[29,30]$ and for the leading $(1,0)$ term at genus-two (the $\partial^{4} \mathcal{R}^{4}$ term).

The leading terms in (3.11) and (3.12), the genus-zero parts $(h=0)$ are of order $y_{D}^{-1}$ and since their coefficients do not depend on the compactification torus they are the same for all $D$. The genus-one $(h=1)$ amplitude is of order $y_{D}^{0}$ and is given by the integral over the complex structure of the world-sheet torus, $\tau$, of the form

$$
I_{1}^{(d)}\left[j_{1}^{(p, q)}\right]:=\int_{\mathcal{F}_{S L(2, \mathbb{Z})}} \frac{d^{2} \tau}{\tau_{2}^{2}} j_{1}^{(p, q)}(\tau)\left(\Gamma_{(d, d)}(\tau)-V_{d}\right)
$$

where $\mathcal{F}_{\mathrm{SL}(2, \mathbb{Z})}$ is a fundamental domain for $\mathrm{SL}(2, \mathbb{Z})$ and $V_{d}=\sqrt{\operatorname{det} G}$ is the volume of $\mathcal{T}^{d}$, and $G_{i j}$ is its metric: $G_{i j}=g_{i j}+b_{i j}$ with $g_{i j}$ a $d \times d$ symmetric square matrix and $b_{i j}$ is a $d \times d$ antisymmetric square matrix. The lattice factor is defined by

$$
\Gamma_{(d, d)}(\tau)=V_{d} \sum_{\left(m^{i}, n^{i}\right) \in \mathbb{Z}^{2 d}} \mathrm{e}^{-\frac{\pi}{\tau_{2}}\left(m^{i}-n^{i} \tau\right) G_{i j}\left(m^{j}-n^{j} \bar{\tau}\right)},
$$


where repeated indices are summed. Thus, for the $\mathcal{R}^{4}$ term with coefficient $\mathcal{E}_{(0,0)}^{(D)}$, since $j_{1}^{(0,0)}=1$ it follows that $[29,30]$

$$
I_{1}^{(d)}\left[j_{1}^{(0,0)}\right]=\frac{\Gamma\left(\frac{d}{2}\right)}{\pi^{\frac{d}{2}}} \mathbf{E}_{[10 \cdots 0] ; \frac{d}{2}-1}^{\mathrm{SO}(d, d)}
$$

which matches the $y_{D}$-independent term on the right-hand side of (3.11). The $\mathrm{SO}(d, d)$ series will be defined in terms of explicit lattice sums in (3.27) (see [4, Appendix C] for details of these series).

For the $\partial^{4} \mathcal{R}^{4}$ term with coefficient $\mathcal{E}_{(1,0)}^{(D)}$, since $j_{1}^{(1,0)}=\mathbf{E}_{2}^{S L(2)}(\tau) /(4 \pi)^{2}$ it follows that $[29,30]$

$$
I_{1}^{(d)}\left[j_{1}^{(1,0)}\right]=\frac{2 \zeta(4) \Gamma\left(\frac{d}{2}+1\right)}{8 \pi^{\frac{d}{2}+1}} \mathbf{E}_{[10 \cdots 0] ; \frac{d}{2}+1}^{\mathrm{SO}(d, d)} .
$$

In the genus-two case, the functions $j_{2}^{(p, q)}$ that enter into the expression $I_{2}\left[j_{2}^{(p, q)}\right]$ have not been determined beyond the leading term, which is simply $j_{2}^{(1,0)}=1$. This contributes to the $\sigma_{2} \mathcal{R}^{4}$ coefficient, $\mathcal{E}_{(1,0)}^{(D)}$, which is given by the integral of the genus two lattice factor $\Gamma_{(d, d)}(\Omega)$

$$
I_{2}^{(d)}\left[j_{2}^{(1,0)}\right]:=\int_{\mathcal{F}_{S p(2, \mathbb{Z})}} \frac{\left|d^{3} \Omega\right|^{2}}{(\operatorname{det} \operatorname{Im} \Omega)^{3}} \Gamma_{(d, d)}(\Omega)
$$

where $\Omega$ is the genus two-period matrix and $\mathcal{F}_{S p(2, \mathbb{Z})}$ is a Siegel fundamental domain for $S p(2, \mathbb{Z})$ and $\Gamma_{(d, d)}(\Omega)$ is the lattice sum defined by

$$
\Gamma_{(d, d)}(\Omega)=\left(V_{d}\right)^{2} \sum_{\left(m_{a}^{i}, n^{i a}\right) \in \mathbb{Z}^{4 d}} e^{-\pi G_{i j}\left(m_{a}^{i}-\Omega_{a b} n^{i b}\right)\left(\operatorname{Im} \Omega^{-1}\right)^{a c}\left(m_{c}^{j}-\Omega_{c d} n^{j d}\right)}
$$

Using results from $[4,13]$, it is possible to deduce from $(3.17)$ that

$$
I_{2}^{(d)}\left[j_{2}^{(1,0)}\right]=\frac{1}{6 \pi}\left(\mathbf{E}_{[0 \cdots 010] ; 2}^{\mathrm{SO}(d, d)}+\mathbf{E}_{[0 \cdots 01] ; 2}^{\mathrm{SO}(d, d)}\right)
$$

for $d \leq 4$, and

$$
I_{2}^{(d)}\left[j_{2}^{(1,0)}\right]=\frac{1}{3 \pi} \mathbf{E}_{[0 \cdots 01] ; 2}^{\mathrm{SO}(d, d)}
$$

for $d \geq 5$. In order to compare these perturbative string theory results with the constant term in (3.12) we need to use the following relations between 
the $\operatorname{SO}(d, d)$ series

$$
\begin{gathered}
\mathbf{E}_{[0 \cdots 010] ; 2}^{\mathrm{SO}(d, d)}=\mathbf{E}_{[0 \cdots 01] ; 2}^{\mathrm{SO}(d, d)}, \\
\mathbf{E}_{[0 \cdots 010] ; d-3}^{\mathrm{SO}(d, d)}=\mathbf{E}_{[0 \cdots 01] ; d-3}^{\mathrm{SO}(d, d)}, \\
\mathbf{E}_{[0 \cdots 01] ; 2}^{\mathrm{SO}(d, d)} \propto \mathbf{E}_{[0 \cdots 10] ; d-3}^{\mathrm{SO}(d, d)}
\end{gathered}
$$

The last equation is a direct consequence of the functional Equations (2.20) for the $\mathrm{SO}(d, d)$ Eisenstein series. These string theory results are in accord with the term linear in $y_{D}$ in the constant term (3.11).

\section{(iii) Semi-classical M-theory}

This is the limit associated with the parabolic subgroup $P_{\alpha_{2}}$. In this limit the volume $\mathcal{V}_{d+1} \rightarrow \infty$ of the M-theory torus becomes large and the semi-classical, or Feynman diagram, approximation to 11-dimensional supergravity is useful. The constant term of the coefficients in (3.1) and (3.2) in this parabolic subgroup is given by (using the relation $\ell_{D}^{D-2}=\ell_{11}^{9} / \mathcal{V}_{d+1}$, as well as $r^{1+d}=\left(\mathcal{V}_{d+1} / \ell_{11}^{d+1}\right)^{3 / 2}$ with $\left.d=10-D\right)[4,7,8,31]$,

$$
\ell_{D}^{8-D} \int_{P_{\alpha_{2}}} \mathcal{E}_{(0,0)}^{(D)} \simeq \frac{\mathcal{V}_{d+1}}{\ell_{11}^{3}}\left(4 \zeta(2)+\left(\frac{\ell_{11}^{d+1}}{\mathcal{V}_{d+1}}\right)^{\frac{3}{d+1}} \mathbf{E}_{\left[10^{d-1}\right] ; \frac{3}{2}}^{\mathrm{SL}(d+1)}\right)
$$

and

$$
\begin{aligned}
\int_{P_{\alpha_{d}}} \mathcal{E}_{(1,0)}^{(D)} \simeq & \frac{\ell_{11} \mathcal{V}_{d+1}}{\ell_{D}^{12-D}}\left(\left(\frac{\mathcal{V}_{d+1}}{\ell_{11}^{d+1}}\right)^{\frac{1}{d+1}} \mathbf{E}_{\left[10^{d-1}\right] ;-\frac{1}{2}}^{\mathrm{SL}(d+1)}+\left(\frac{\ell_{11}^{d+1}}{\mathcal{V}_{d+1}}\right)^{\frac{5}{d+1}} \mathbf{E}_{\left[10^{d-1}\right] ; \frac{5}{2}}^{\mathrm{SL}(d+1)}\right. \\
& \left.+\left(\frac{\ell_{11}^{d+1}}{\mathcal{V}_{d+1}}\right)^{\frac{8}{d+1}} \mathbf{E}_{\left[010^{d-2} 10\right] ; 2}^{\mathrm{SL}(d+1)}\right) .
\end{aligned}
$$

The precise values of the constants are given in tables A.4 and A.8.

The various contributions in (3.22) agree with the expressions obtained by evaluating the sum of one-loop and two-loop Feynman diagram contributions to the amplitude in 11-dimensional supergravity compactified on a $(d+1)$-torus $[7,8,31]$. The two terms in the $\mathcal{R}^{4}$ coefficient $(3.22)$ arise from the compactified one-loop diagrams together with the counterterm diagram as in [7], while the terms in $\partial^{4} \mathcal{R}^{4}$ coefficient (3.23) arise from the sum of the compactified two-loop diagrams and the one-loop diagram that includes a vertex for the one-loop counterterm [31]. We refer to [4, Appendices B and G] 
for the precise connection between these computations and the $\mathrm{SL}(d+1)$ series entering in (3.23).

\subsection{Bifurcation of $\mathcal{E}_{(1,0)}^{(D)}$ between $D=5$ and $D=6$}

The decompactification of the $D=5$ coefficient $\mathcal{E}_{(1,0)}^{(5)}$,

$$
\mathcal{E}_{(1,0)}^{(5)}=\frac{1}{2} \mathbf{E}_{[100000] ; \frac{5}{2}}^{E_{6}}
$$

merits special discussion. As noted earlier, the third line of table A.6 shows that the constant term of the $E_{6}$ Eisenstein series, corresponding to the decompactification from $D=5$ to $D=6$ results in the sum of two $\operatorname{SO}(5,5)$ series in the combination $\frac{1}{2} \hat{\mathbf{E}}_{[10000] ; \frac{5}{2}}^{\mathrm{SO}(5,5)}+\frac{4}{45} \hat{\mathbf{E}}_{[00001] ; 3}^{\mathrm{SO}(5,5)}$ mutiplying $r^{20 / 3}$, where the hats indicate the finite part of the series after subtraction of an $\epsilon$ pole as in [4]. Although the individual $\mathrm{SO}(5,5)$ series have poles in $s$, the residues of these poles cancel and the sum is finite (as discussed in $[4,5]$ ).

This is seen by using the relations $\ell_{5}^{3}=\ell_{6}^{4} / r_{5}$ and $r=\left(r_{5} / \ell_{6}\right)^{1 / 2}$, leading to

$$
\begin{aligned}
\int_{P_{\alpha_{6}}} \mathcal{E}_{(1,0)}^{(5)}= & \frac{\ell_{6}^{6} r_{5}}{\ell_{5}^{7}}\left(\frac{1}{2} \hat{\mathbf{E}}_{[10000] ; \frac{5}{2}}^{\mathrm{SO}(5,5)}+\frac{4}{45} \hat{\mathbf{E}}_{[00001] ; 3}^{\mathrm{SO}(5,5)}\right. \\
& \left.+2 \log \left(\frac{r_{5}}{\ell_{6} \mu}\right) \mathcal{E}_{(0,0)}^{(6)}+\frac{\zeta(7)}{6}\left(\frac{r_{5}}{\ell_{6}}\right)^{6}\right) .
\end{aligned}
$$

where $\mu$ is a constant scale factor. The term linear in $r_{5}$ is the one that multiplies the $D=6$ coefficient, $\mathcal{E}_{(1,0)}^{(6)}$ so that

$$
\mathcal{E}_{(1,0)}^{(6)}=\frac{1}{2} \hat{\mathbf{E}}_{[10000] ; \frac{5}{2}}^{\mathrm{SO}(5,5)}+\frac{4}{45} \hat{\mathbf{E}}_{[00001] ; 3}^{\mathrm{SO}(5,5)} .
$$

In order to interpret the $\log r_{5}$ term in (3.25) we need to analyze properties of the $\mathrm{SO}(5,5)$ series in this expression. Although such properties are contained in the general expressions in section 2, it is illuminating to obtain them from the representation of such series as lattice sums. In general, such lattice sums are awkward to analyze but in this case the expression for $\mathbf{E}_{\left[10^{d-1}\right] ; s}^{\mathrm{SO}(\text { given }}$ in (C.2) of [4]) is expressible in a useful manner. The expression given in [4] is a provided by a Siegel-Weil formula [32] relating the integral over the moduli space of genus-one Riemann surfaces of $\mathrm{SL}(2, \mathbb{Z})$ Eisenstein series 
times lattice sums and $\mathrm{SO}(d, d)$ Eisenstein series,

$$
\mathbf{E}_{\left[10^{d-1}\right] ; s}^{\mathrm{SO}(d, d)}=\frac{\pi^{s}}{2 \zeta(2 s+2-d) \Gamma(s)} \int_{\mathcal{F}_{S L(2, \mathbb{Z})}} \frac{d^{2} \tau}{\tau_{2}^{2}} \mathbf{E}_{s+1-\frac{d}{2}}(\tau)\left(\Gamma_{(d, d)}(\tau)-V_{d}\right),
$$

where $\mathbf{E}_{s}(\tau)=\sum_{(m, n) \neq(0,0)} y^{s} /|m+n \tau|^{2 s}$ is the usual $\operatorname{SL}(2, \mathbb{Z})$ Eisenstein series and $\Gamma_{(d, d)}(\tau)$ is defined in (3.14). It follows from this definition that the series satisfies the functional equation

$$
\mathbf{E}_{\left[10^{d-1}\right] ; s}^{\mathrm{SO}(d)}=\frac{\xi(2 s-2 d+3) \xi(2 s-d+1)}{\xi(2 s) \xi(2 s-d+2)} \frac{\zeta(2 s)}{\zeta(2 d-2-2 s)} \mathbf{E}_{\left[10^{d-1}\right] ; d-1-s}^{\mathrm{SO}(d, d)},
$$

where $\xi(s)$ is the completed Riemann $\zeta$-function $\xi(s)=\pi^{-s / 2} \Gamma\left(\frac{s}{2}\right) \zeta(s)$. This functional relation implies that the $\mathrm{SO}(d, d)$ series has a single pole at $s=d / 2$

$$
\mathbf{E}_{\left[10^{d-1}\right] ; \frac{d}{2}+\epsilon}^{\mathrm{SO}(d, d)}=\frac{6}{d-2} \frac{\mathbf{E}_{\left[10^{d-1}\right] ; \frac{d}{2}-1}^{\mathrm{SO}(d, d)}}{\epsilon}+\hat{\mathbf{E}}_{\left[10^{d-1}\right] ; \frac{d}{2}}^{\mathrm{SO}(d, d)}+O(\epsilon)
$$

The residue of the series $\mathbf{E}_{[0 \cdots 01] ; s}^{\mathrm{SO}(d, d)}$ at $s=(d+1) / 2$ can be extracted using the methods of section 2 .

For the $\mathrm{SO}(5,5)$ case this becomes

$$
\mathbf{E}_{[10000] ; \frac{5}{2}+\epsilon}^{\mathrm{SO}(5,5)}=\frac{2 \mathbf{E}_{[10000] ; \frac{3}{2}}^{\mathrm{SO}(5,5)}}{\epsilon}+\hat{\mathbf{E}}_{[10000] ; \frac{5}{2}}^{\mathrm{SO}(5,5)}+O(\epsilon)
$$

The general methods of section 2 indicates that the series $\mathbf{E}_{[00001] ; s}^{\mathrm{SO}(5,5)}$ has a single pole at $s=3$ given by

$$
\mathbf{E}_{[00001] ; 3+\epsilon}^{\mathrm{SO}(5,5)}=\frac{45}{4} \frac{\mathbf{E}_{[10000] ; \frac{3}{2}}^{\mathrm{SO}(5,5)}}{\epsilon}+\hat{\mathbf{E}}_{[00001] ; 3}^{\mathrm{SO}(5,5)}+O(\epsilon) .
$$

It is striking that the residue of the pole is given by the $\mathcal{R}^{4}$ coefficient, $\mathcal{E}_{(0,0)}^{(6)}=\mathbf{E}_{[10000] ; 3 / 2}^{\mathrm{SO}(5,5)}$. This is the reason why the coefficient of the $r_{5} \log \left(r_{5} / \ell_{6}\right)$ term in $(3.25)$ is the $\mathcal{E}_{(0,0)}^{(6)}$ coefficient (see [5, equation $\left.(2.11)\right]$ with $D=5$ ). 
Because the residues of the $\epsilon$ poles in (3.30) and (3.31) are both proportional to $\mathbf{E}_{[10000] ; \frac{3}{2}}^{\mathrm{SO}(5,5)}$ the $\partial^{4} \mathcal{R}^{4}$ coefficient in $D=6$ can be written as the limit

$$
\mathcal{E}_{(1,0)}^{(6)}=\frac{1}{2} \lim _{\epsilon \rightarrow 0}\left(\mathbf{E}_{[10000] ; \frac{5}{2}+\epsilon}^{\mathrm{SO}(5,5)}+\frac{8}{45} \mathbf{E}_{[00001] ; 3-\epsilon}^{\mathrm{SO}(5,5)}\right)
$$

as suggested in [4]. The cancellation of divergences is a general feature of all coefficient functions for all values of $D$ and is consistent with the absence of ultraviolet divergences in string theory.

The coefficient $\mathcal{E}_{(1,0)}^{(D)}$ for $5<D<10$ continues to be given by the sum of two distinct series [4] and it is only for the exceptional groups and $\mathrm{SL}(2, \mathbb{Z})$ that this coefficient is given by a single Eisenstein series.

\section{Constant terms for the solution of the $E_{8}$ inhomogeneous Laplace eigenvalue equation}

The previous sections concern the coefficients in the effective action that satisfy the Laplace eigenvalue equations (1.4) and (1.5) with solutions that are Eisenstein series, whereas the coefficient $\mathcal{E}_{(0,1)}^{(D)}$ of the $\partial^{6} \mathcal{R}^{4}$ coefficient in (3.3) satisfies the inhomogeneous equation (1.6). Its solution, subject to appropriate boundary conditions, is formally given in terms of a Green function by (for $D \neq 6$ )

$$
\mathcal{E}_{(0,1)}^{(D)}=\mathcal{E}_{\text {hom }}^{(D)}-\left(\Delta^{(D)}-\frac{6(14-D)(D-6)}{D-2}\right)^{-1}\left(\mathcal{E}_{(0,0)}^{(D)}\right)^{2},
$$

where $\mathcal{E}_{\text {hom }}^{(D)}$ is a solution to the homogeneous equation. Expressing this solution in a more explicit manner is a challenge which we will not undertake here (see [9] for a discussion of the $\operatorname{SL}(2, \mathbb{Z})$ case). Instead, we will study the appropriate constant terms of this coefficient in two of the parabolic subgroups, $P_{\alpha_{1}}$ and $P_{\alpha_{d+1}}$, of relevance in this paper, as was done for the cases $6 \leq D \leq 10$ (i.e., for duality groups of rank $r \leq 5$ ) in $[4,5,9,12]$. We shall omit the analysis of the third subgroup $P_{\alpha_{2}}$ for economy of space, since it presents few new issues. Furthermore, for the sake of brevity, rather than considering all the remaining cases $(D=3,4,5)$, we will consider the fundamental example of the $D=3$ coefficient, $\mathcal{E}_{(0,1)}^{(3)}$, from which the others can be obtained. This is an $E_{8}$ automorphic function that satisfies the inhomogeneous Laplace eigenvalue equation (1.6):

$$
\left(\Delta^{(3)}+198\right) \mathcal{E}_{(0,1)}^{(3)}=-\left(\mathcal{E}_{(0,0)}^{(3)}\right)^{2},
$$


where the source term involves the square of

$$
\mathcal{E}_{(0,0)}^{(3)}=\mathbf{E}_{[10000000] ; \frac{3}{2}}^{E_{8}} .
$$

As we will see, the requirement that these constant terms have the correct structure once again determines them.

We note that the issue of whether the solution (4.1) is unique depends on whether there is a solution to the homogeneous equation with vanishing constant terms in the parabolic subgroups $P_{\alpha_{d+1}}$ and $P_{\alpha_{1}}$ (limits (i), (ii)) considered in this section. Such a solution would be an automorphic function with eigenvalue -198 . We have verified that that no maximal parabolic Eisenstein series satisfies the boundary conditions in either of the two limits under consideration, but it is possible (but seems unlikely) that some more general series with the same eigenvalue may satisfy them. There are also potentially cusp form solutions to the homogeneous equation, though these also seem unlikely to exist because of the reasons mentioned in the Introduction. Their presence would amount to an ambiguity in the solution.

\section{(i) Decompactification to $D=4$}

Before specializing to $D=3$ (the $E_{8}$ case) we will review the behaviour of $\mathcal{E}_{(0,1)}^{(D)}$ for general $D=10-d$ in this limit. Based on input from string theory the constant term in the parabolic subgroup $P_{\alpha_{d+1}}$, the decompactification limit, should consist of five components with distinct powers of $r$ (see $[5$, equation (2.12)]),

$$
\begin{aligned}
\int_{P_{\alpha_{d}+1}} \mathcal{E}_{(0,1)}^{(D)} \simeq & \left(\frac{\ell_{D+1}}{\ell_{D}}\right)^{14-D}\left(\frac{r_{d}}{\ell_{D+1}} \mathcal{E}_{(0,1)}^{(D+1)}+\left(\frac{r_{d}}{\ell_{D+1}}\right)^{14-D}\right. \\
& +\left(\frac{r_{d}}{\ell_{D+1}}\right)^{8-D} \mathcal{E}_{(0,0)}^{(D+1)}+\left(\frac{r_{d}}{\ell_{D+1}}\right)^{4-D} \mathcal{E}_{(1,0)}^{(D+1)} \\
& \left.+\left(\frac{r_{d}}{\ell_{D+1}}\right)^{15-2 D}+O\left(\mathrm{e}^{-r_{d} / \ell_{D+1}}\right)\right)
\end{aligned}
$$

(certain $\log r$ factors that arise for specific values of $D$ have been suppressed in this expression). The following is a sketchy summary of the interpretation of the five components of this expression. The term proportional to $r_{d}$ contains the coefficient $\mathcal{E}_{(1,0)}^{(D+1)}$ in the decompactified theory. The second term, which has a constant coefficient, is the $n=3$ contribution to the infinite series of $\left(s r_{d}^{2}\right)^{n} \mathcal{R}^{4}$ terms $(n \geq 0)$ that generates the one-loop supergravity threshold behaviour in $D+1$ dimensions (the first two terms arose in (3.9) 
and (3.10)). Similarly, the third term, proportional to $\mathcal{E}_{(0,0)}^{(D+1)}$, is the $n=1$ term in a series of terms of the form $r_{d}^{2-D}\left(s r_{d}^{2}\right)^{n+2} \mathcal{R}^{4}$ that contributes to the second threshold in $D+1$ dimensions (the $n=0$ term arose in (3.10)). The fourth term, proportional to $\mathcal{E}_{(1,0)}^{(D+1)}$, is the $n=0$ term in a new infinite series of terms of the form $r_{d}^{-(2+D)}\left(s r_{d}^{2}\right)^{n+3} \mathcal{R}^{4}$ that generates the correct $(D+1)$-dimensional behaviour of a new threshold contribution at order $\ell_{D}^{8}$. Finally, the last term in parentheses is the $n=0$ term in a series of terms of the form $r_{d}^{-2 D+9}\left(s r_{d}^{2}\right)^{n+3} \mathcal{R}^{4}$, which sums to the two-loop supergravity threshold in $D+1$ dimensions. Again the structure of these terms that contribute to thresholds is in accord with unitarity, generalizing the discussion in $[28]$.

Thus, as stated earlier, the constant term of the series $\mathcal{E}_{(0,1)}^{(D)}$ in this parabolic subgroup contains the information concerning all three coefficients in $D+1$ dimensions.

We now consider the constant term that arises from the solution of (4.2), which describes limit (i) in the $E_{8}$ case. We are interested in the limit associated with the parabolic subgroup $P_{\alpha_{8}}$, associated with the right-hand node of the $E_{8}$ Dynkin diagram of figure 1 . As a template for what this constant term should look like, we note that the constant term of the source term can be expressed as

$$
\begin{aligned}
\int_{P_{\alpha_{8}}}\left(\mathcal{E}_{(0,0)}^{(3)}\right)^{2}= & \frac{\ell_{4}^{11}}{\ell_{3}^{11}}\left(\frac{r_{7}}{\ell_{4}}\left(\mathbf{E}_{\left[10^{6}\right] ; \frac{3}{2}}^{E_{7}}\right)^{2}+\left(\frac{r_{7}}{\ell_{4}}\right)^{5} \frac{6 \zeta(5)}{\pi} \mathbf{E}_{\left[10^{6}\right] ; \frac{3}{2}}^{E_{7}}\right. \\
& \left.+\left(\frac{r_{7}}{\ell_{4}}\right)^{9} \frac{9 \zeta(5)^{2}}{\pi^{2}}+O\left(\mathrm{e}^{-r_{7} / \ell_{4}}\right)\right) .
\end{aligned}
$$

Based on the structure of this expression, together with the form anticipated in (4.4) with $D=3$, we make the ansatz

$$
\begin{aligned}
\int_{P_{\alpha_{8}}} \mathcal{E}_{(0,1)}^{(3)}= & \frac{\ell_{4}^{11}}{\ell_{3}^{11}}\left(\frac{r_{7}}{\ell_{4}} F_{1}^{E_{7}}+\frac{r_{7}}{\ell_{4}} \log \left(\frac{r_{7}}{\ell_{4} \mu}\right) F_{2}^{E_{7}}+\left(\frac{r_{7}}{\ell_{4}}\right)^{5} F_{3}^{E_{7}}\right. \\
& \left.+\left(\frac{r_{7}}{\ell_{4}}\right)^{9} F_{4}^{E_{7}}+\left(\frac{r_{7}}{\ell_{4}}\right)^{11} F_{5}^{E_{7}}+O\left(\mathrm{e}^{-r_{7} / \ell_{4}}\right)\right)
\end{aligned}
$$

where $\mu$ is a constant scale factor in the logarithm (the presence of which will become clear later) and the coefficients $F_{r}^{E_{7}}$ are coefficients that will now be determined.

In the limit corresponding to this parabolic the Laplacian $\Delta^{(3)}$ on $E_{8} / \mathrm{SO}(16)$ decomposes into a sum of the Laplacian $\Delta^{(4)}$ on $E_{7} / \mathrm{SU}(8)$ and 
the Laplacian along the $r_{7}$ direction (as in [4, Appendix H.2]),

$$
\Delta^{(3)} \rightarrow \Delta^{(4)}+\frac{1}{4}\left(r_{7} \partial_{r_{7}}\right)^{2}-\frac{29}{2} r_{7} \partial_{r_{7}}
$$

Substituting this expression together with (4.5) and (4.6) into (4.2) (and using $\left.\ell_{3}=\ell_{4}^{2} / r_{7}\right)$ suggests matching terms as follows:

$$
\begin{aligned}
\left(\Delta^{(4)}+60\right) F_{1}^{E_{7}} & =-\left(\mathbf{E}_{\left[10^{6}\right] ; \frac{3}{2}}^{E_{7}}\right)^{2}, \\
\left(\Delta^{(4)}+60\right) F_{2}^{E_{7}} & =0, \\
\left(\Delta^{(4)}+30\right) F_{3}^{E_{7}} & =-\frac{6 \zeta(5)}{\pi} \mathbf{E}_{\left[10^{6}\right] ; \frac{3}{2}}^{E_{7}}, \\
\left(\Delta^{(4)}+8\right) F_{4}^{E_{7}} & =-\frac{9 \zeta(5)^{2}}{\pi^{2}}, \\
\Delta^{(4)} F_{5}^{E_{7}} & =0 .
\end{aligned}
$$

In deriving these equations the relations

$$
\left(\frac{1}{4}\left(r_{7} \partial_{r_{7}}\right)^{2}-\frac{29}{2} r_{7} \partial_{r_{7}}+198\right) r_{7}^{22}=0,
$$

and

$$
\left(\frac{1}{4}\left(r_{7} \partial_{r_{7}}\right)^{2}-\frac{29}{2} r_{7} \partial_{r_{7}}+138\right) r_{7}^{12} \log \left(r_{7}\right)=-\frac{17}{2} r_{7}^{12}
$$

were used, which accounts for the $\log r_{7}$ factor in (4.6). Equation (4.8) is the equation satisfied by the $\partial^{6} \mathcal{R}^{4}$ coefficient in four dimensions ((1.6) with $D=4)$, so we identify

$$
F_{1}^{E_{7}}=\mathcal{E}_{(0,1)}^{(4)}
$$

This uses the fact that the right-hand side of (4.8) involves the square of the $D=4 \mathcal{R}^{4}$ coefficient,

$$
\mathcal{E}_{(0,0)}^{(4)}=\mathbf{E}_{\left[10^{6}\right] ; \frac{3}{2}}^{E_{7}} .
$$

In principle, we could repeat this procedure and study the constant term of the $E_{7}$ coefficient in the parabolic subgroup $P\left(\alpha_{7}\right)$, and so on, in order to match with the known coefficients for the lower-rank cases. Equation (4.9) 
is solved by the maximal parabolic Eisenstein series that is proportional to the $\partial^{4} \mathcal{R}^{4}$ coefficient in four dimensions,

$$
F_{2}^{E_{7}} \propto \mathbf{E}_{\left[10^{6}\right] ; \frac{5}{2}}^{E_{7}} \propto \mathcal{E}_{(1,0)}^{(4)}
$$

as required by the general formula (4.4) for $D=3$. The discussion of the $\partial^{4} \mathcal{R}^{4}$ interaction in section 3 demonstrates that this satisfies the appropriate boundary conditions. Equation (4.10) is solved by

$$
F_{3}^{E_{7}}=\frac{\zeta(5)}{2 \pi} \mathbf{E}_{\left[10^{6}\right] ; \frac{3}{2}}^{E_{7}}
$$

which is proportional to $\mathcal{E}_{(0,0)}^{(4)}$ given in $(3.6)$, as it should be according to (4.4). In the case of (4.11) none of the solutions of the homogenous equation

$$
\left(\Delta^{(4)}+8\right) F_{4}^{E_{7}}=0
$$

are compatible with the boundary conditions imposed by string perturbation. Therefore the solution to (4.11) is the constant function

$$
F_{4}^{E_{7}}=-\frac{9 \zeta(5)^{2}}{8 \pi^{2}} .
$$

Extracting the value of $F_{5}^{E_{7}}$, which satisfies (4.12), is more subtle since the constant term expansion of the source term in (4.5) does not include an explicit power of $r^{11}$. Nevertheless, the full solution, $\left(\Delta^{(3)}+198\right)^{-1}\left(\mathcal{E}_{(0,0)}^{(3)}\right)^{2}$, does contain such a contribution in its zero mode expansion that can be extracted by projecting this solution onto a solution of the homogeneous equation, as in $[9$, section 5.4]. This gives rise to a constant solution,

$$
F_{5}^{E_{7}}=\text { const. }
$$

Although the values of the constants in (4.17) and (4.21) can be extracted from the complete solution using an extension of the methods in [9], this will not be carried out here.

In summary, we have determined the functions $F_{1}^{E_{7}}, \ldots, F_{5}^{E_{7}}$ that are the power-behaved components of the constant term $\int_{P_{\alpha_{8}}} \mathcal{E}_{(0,1)}^{(3)}$ defined by (4.6), and this matches the form (4.4) anticipated from string theory considerations. Strikingly, this constant term contains all three coefficients for the $E_{7}$ case, $\mathcal{E}_{(0,0)}^{(4)}, \mathcal{E}_{(1,0)}^{(4)}, \mathcal{E}_{(0,1)}^{(4)}$, as is evident from (4.4) with $D=3$. Therefore 
all the preceding coefficients of the lower rank examples are contained in this one example.

\section{(ii) The perturbative expansion}

The perturbative expansion is given by the constant term associated with the maximal parabolic subgroup associated with the node $P_{\alpha_{1}}$ with Levi subgroup $\mathrm{GL}(1) \times \mathrm{SO}(7,7)$. String perturbation theory (an expansion in powers of $y_{3}$ starting at $\left.y_{3}^{-1}\right)$ requires this to have the form

$$
\ell_{3}^{11} \int_{P_{\alpha_{1}}} \mathcal{E}_{(0,1)}^{(3)}=\ell_{s}^{11}\left(\sum_{k=0}^{3} y_{3}{ }^{k-1} F_{k}^{\mathrm{SO}(7,7)}+\mathrm{O}\left(\mathrm{e}^{-1 / y_{3}}\right)\right) .
$$

The coefficients $F_{k}^{\mathrm{SO}(7,7)}$ can be determined by a procedure analogous to the one in the previous limit, as follows. First the Laplacian $\Delta^{(3)}$ on $E_{8} / \mathrm{SO}(16)$ is decomposed in this limit into a sum of the Laplacian on $\mathrm{SO}(7,7) /(\mathrm{SO}(7) \times \mathrm{SO}(7))$ and a Laplacian along the $y_{3}$ direction $^{5}$

$$
\Delta^{(3)} \rightarrow \Delta^{\mathrm{SO}(7,7) /(\mathrm{SO}(7) \times \mathrm{SO}(7))}+\frac{1}{2}\left(y_{3} \partial_{y_{3}}\right)^{2}+23 y_{3} \partial_{y_{3}} .
$$

Next, the constant term of the source is obtained by substituting the expansion of $\mathcal{E}_{(0,0)}^{(3)}$ (given in table A.4 with $r^{-4}=y_{3}$ ), resulting in

$$
\int_{P_{\alpha_{1}}}\left(\mathcal{E}_{(0,0)}^{(3)}\right)^{2}=\frac{\ell_{s}^{11}}{\ell_{3}^{11}}\left(\frac{4 \zeta(3)^{2}}{y_{3}}+\frac{6}{\pi} \mathbf{E}_{\left[10^{6}\right] ; \frac{3}{2}}^{\mathrm{SO}(7,7)}+\frac{9 y_{3}}{4 \pi^{2}}\left(\mathbf{E}_{\left[10^{6}\right] ; \frac{3}{2}}^{\mathrm{SO}(7,7)}\right)^{2}+O\left(\mathrm{e}^{-1 / y_{3}}\right)\right) .
$$

The structure of this expression is consistent with (4.22), which we may use as an ansatz for the solution. Substituting (4.22), (4.23) and (4.24) into (4.2) results in equations that determine the coefficients $F_{k}$ (using $\ell_{3}=\ell_{s} y_{3}$ ),

$$
\begin{aligned}
\left(\Delta^{\mathrm{SO}(7,7) / \mathrm{SO}(7) \times \mathrm{SO}(7)}-6\right) F_{0}^{\mathrm{SO}(7,7)} & =-4 \zeta(3)^{2}, \\
\left(\Delta^{\mathrm{SO}(7,7) / \mathrm{SO}(7) \times \mathrm{SO}(7)}+\frac{11}{2}\right) F_{1}^{\mathrm{SO}(7,7)} & =-\frac{6 \zeta(3)}{\pi} \mathbf{E}_{\left[10^{6}\right] ; \frac{5}{2}}^{\mathrm{SO}(7,7)}
\end{aligned}
$$

${ }^{5}$ Requiring the tree-level contributions to $\mathcal{R}^{4}$ and $\partial^{4} \mathcal{R}^{4}$ to be annihilated by the $\mathrm{SO}(d, d) /(\mathrm{SO}(d) \times \mathrm{SO}(d))$ Laplacian implies

$$
\Delta^{(D)} \rightarrow \Delta^{\mathrm{SO}(d, d) /(\mathrm{SO}(d) \times \mathrm{SO}(d))}+\frac{D-2}{2}\left(y_{D} \partial_{y_{D}}\right)^{2}+\frac{D^{2}-19 D+94}{2} y_{D} \partial_{y_{D}} .
$$




$$
\begin{aligned}
& \left(\Delta^{\mathrm{SO}(7,7) / \mathrm{SO}(7) \times \mathrm{SO}(7)}+18\right) F_{2}^{\mathrm{SO}(7,7)}=-\frac{9}{4 \pi^{2}}\left(\mathbf{E}_{\left[10^{6}\right] ; \frac{5}{2}}^{\mathrm{SO}(7,7)}\right)^{2}, \\
& \left(\Delta^{\mathrm{SO}(7,7) / \mathrm{SO}(7) \times \mathrm{SO}(7)}+\frac{63}{2}\right) F_{3}^{\mathrm{SO}(7,7)}=0 .
\end{aligned}
$$

A solution to (4.25) that is compatible with string perturbation theory is the constant

$$
F_{0}^{\mathrm{SO}(7,7)}=\frac{2 \zeta(3)}{3},
$$

which is precisely the genus zero (tree-level) contribution [4]. A solution to the homogeneous equation $((4.26)$ with no source term) that is consistent with string theory is $\mathbf{E}_{\left[10^{6}\right] ; \frac{11}{2}}^{\mathrm{SO}(7,7)}$, resulting in a solution of (4.26) given by

$$
F_{1}^{\mathrm{SO}(7,7)}=\frac{1}{12} \mathbf{E}_{\left[10^{6}\right] ; \frac{11}{2}}^{\mathrm{SO}(7,7)}+\frac{\zeta(3)}{2 \pi} \mathbf{E}_{\left[10^{6}\right] ; \frac{5}{2}}^{\mathrm{SO}(7,7)} .
$$

This agrees with the genus one string theory expression $I_{1}^{(d)}\left[j_{1}^{(0,1)}\right]$ evaluated in $\left[4\right.$, Appendix D] using $j_{1}^{(0,1)}=10 \mathbf{E}_{3}(\tau) /(4 \pi)^{3}+\zeta(3) / 32$. The function $F_{2}^{\mathrm{SO}(7,7)}$ satisfies the inhomogeneous Laplace eigenvalue equation (4.27), which may be analysed in the same manner as analogous examples considered in $[4,5,9,12]$. The function, $F_{3}^{\mathrm{SO}(7,7)}$, satisfies the source-free (homogeneous) equation (4.28) since there is no $y_{3}^{2}$ term in the constant term of the source. As we will see, a solution of relevance to string theory is given by the linear combination of maximal parabolic Eisenstein series,

$$
F_{3}^{\mathrm{SO}(7,7)}=\alpha \mathbf{E}_{\left[0^{6} 1\right] ; 3}^{\mathrm{SO}(7,7)}+\beta \mathbf{E}_{\left[0^{5} 10\right] ; 3}^{\mathrm{SO}(7,7)}+\gamma \mathbf{E}_{\left[0^{2} 10^{4}\right] ; \frac{3}{2}}^{\mathrm{SO}(7,7)}
$$

where $\alpha, \beta$ and $\gamma$ are constants that are determined from the boundary conditions. By a direct evaluation of the series using the methods of section 2 we find that these Eisenstein series satisfy the relations

$$
\begin{aligned}
& \mathbf{E}_{[0000001] ; 3}^{\mathrm{SO}(7,7)}=\mathbf{E}_{[0000010] ; 3}^{\mathrm{SO}(7,7)}, \\
& \mathbf{E}_{[0010000] ; \frac{3}{2}}^{\mathrm{SO}(7,7)}=0 .
\end{aligned}
$$

Therefore the expression (4.31) takes the form

$$
F_{3}^{\mathrm{SO}(7,7)}=(\alpha+\beta) \mathbf{E}_{\left[0^{6} 1\right] ; 3}^{\mathrm{SO}(7,7)}
$$

The normalization is fixed by comparison with the genus three contribution in string theory in the limit in which the volume of the seven-torus, $\mathcal{T}^{7}$, is 
large (see [4, Appendix F]), resulting in

$$
\alpha+\beta=\frac{\operatorname{vol}\left(\mathcal{F}_{S p(3, \mathbb{Z})}\right)}{2 \zeta(6)}=\frac{1}{270},
$$

where $\operatorname{vol}\left(\mathcal{F}_{S p(3, \mathbb{Z})}\right)=\zeta(6) / 135$ is the volume of the Siegel fundamental domain for $S p(3, \mathbb{Z})[33]$.

To summarize, we have determined the constant terms of the solution of Equation (4.2) for $\mathcal{E}_{(0,1)}^{(3)}$ in the parabolic subgroup $P_{\alpha_{1}}$ that agree with the results of the explicit evaluation of string perturbation theory.

\section{Summary and comments}

In this paper we have determined the expressions for the coefficients $\mathcal{E}_{(0,0)}^{(D)}$, $\mathcal{E}_{(1,0)}^{(D)}$ of the $\mathcal{R}^{4}, \partial^{4} \mathcal{R}^{4}$ interactions, the first two higher derivative terms in the low-energy expansion of the four-supergraviton scattering amplitude in maximally supersymmetric string theory. These coefficients are the maximal parabolic Eisenstein series in (3.6) and (3.7) for the duality groups $E_{6}(\mathbb{Z})$, $E_{7}(\mathbb{Z})$ and $E_{8}(\mathbb{Z})$. All the lower rank cases that were determined in earlier work follow from these by considering the behaviour in the vicinity of the decompactification cusp, limit (i), defined by the root $\alpha_{d+1}$. Indeed, starting from the highest-rank case, where the duality group is $E_{8}(\mathbb{Z})$ and $D=3$, all the lower-rank cases are contained in the constant term for the parabolic subgroup defined by the root $\alpha_{8}$. Thus, the constant term in limit (i) leads to a chain of nested finite combinations of maximal parabolic Eisenstein series. In other words, the coefficients of the interactions for the lower rank duality groups can be deduced by successive decompactifications to spacetime dimensions $4 \leq D \leq 10$. Although there might be ambiguities due to the presence of cusp forms, as discussed in the Introduction, it is likely that these are absent for the kinds of series we are considering here. A curious feature arises on decompactifying the five-dimensional $\partial^{4} \mathcal{R}^{4}$ interaction to six dimensions, where a bifurcation arises in which $\mathcal{E}_{(1,0)}^{(5)}$ splits into the sum of two $\mathrm{SO}(5,5)$ Eisenstein series in the $D=6$ theory. Whereas each of these series has a pole at the value $s=5 / 2$, the poles cancel and the sum of the terms is analytic. The same phenomenon has previously been noted in dimensions $D \geq 7$ in [4].

In section 4 we considered the coefficient of the $\partial^{6} \mathcal{R}^{4}$ interaction in the $E_{8}$ case, $\mathcal{E}_{(0,1)}^{(3)}$, which solves the inhomogeneous Laplace eigenvalue equation, (4.2), and is not an Eisenstein series. Although we did not discuss the full solution in this case, the constant term for this coefficient in the parabolic 
subgroup labelled by the root $\alpha_{8}$ was shown to contain all three of the $E_{7}$ automorphic coefficient functions $\mathcal{E}_{(0,0)}^{(4)}, \mathcal{E}_{(1,0)}^{(4)}$ and $\mathcal{E}_{(0,1)}^{(4)}$, and therefore contains within it the complete chain of coefficients in dimensions $4 \leq D \leq 10$, including those determined in earlier work.

Furthermore, we determined the constant terms of these coefficient functions in the parabolic subgroup defined by the root $\alpha_{1}$ that determines the behaviour in limit (ii), the limit of string perturbation theory. In almost all cases these power-behaved terms match the results obtained directly from type II superstring perturbation theory evaluated on higher-dimensional tori, the only exceptions being those cases in which the string calculations have not yet been carried out. Similarly the behaviour of the $\mathcal{R}^{4}$ and $\partial^{4} \mathcal{R}^{4}$ interactions in limit (iii), in which the volume of the $(d+1)$-dimensional $M$-theory torus is large, is contained in the constant terms for the parabolic subgroup defined by the root $\alpha_{2}$. These constant terms precisely match calculations in semiclassical 11-dimensional supergravity based on one- and two-loop Feynman diagrams $[7,8,17,31]$ (the constant term of the $\partial^{6} \mathcal{R}^{4}$ coefficient in this parabolic subgroup has yet to be considered).

These considerations made extensive use of the properties of constant terms in order to match the boundary data obtained from string theory and supergravity. In the case of Eisenstein series the constant terms are power series in the parameter $r$, as defined in section 2.4, though the automorphic function $\mathcal{E}_{(0,1)}^{(D)}$ also contains terms that are exponentially small in the large- $r$ limit (interpreted as instanton anti-instanton pairs with zero net instanton charge). The non-constant terms of the functions we have discussed are also of great interest within string theory since they are interpreted as sums over contributions of charged instantons that are associated with euclidean $p$-branes of various kinds (where $0 \leq p \leq 6$ ) wrapped around $(p+1)$-cycles of the torus, $\mathcal{T}^{d}$. The spectrum of such instantons determines the behaviour of the non-zero Fourier components of the coefficient functions at the cusps. Furthermore, the instanton spectrum in $D$ dimensions $(D=10-d)$ is related to the spectrum of BPS particle states in $D+1$ dimensions, which are charged black holes of various kinds that play an important rôle in the higher-dimensional theory. The fractional BPS nature of the $\mathcal{R}^{4}, \partial^{4} \mathcal{R}^{4}$ and $\partial^{6} \mathcal{R}^{4}$ interactions is encoded in the instanton measure factors that enter the expansion of the coefficients $\mathcal{E}_{(0,0)}^{(D)}, \mathcal{E}_{(1,0)}^{(D)}$ and $\mathcal{E}_{(0,1)}^{(D)}$ near each cusp [34-36]. The 1/8-BPS configurations that enters in $\mathcal{E}_{(0,1)}^{(D)}$ are particularly subtle. It would be interesting to understand their properties more precisely.

We end with some brief comments about other avenues that deserve further exploration: 
There are many other ways in which Eisenstein series and other automorphic forms could enter into the discussion of string theory scattering. Clearly the maximal parabolic series of interest in this paper are but a small subset of the general series. Though we consider it unlikely for the examples here, cusp forms might also contribute to the solutions - this would signify deep arithmetic complexity. Furthermore, a wider variety of constant terms, defined with respect to other maximal and non-maximal parabolic subgroups may well have a role to play in aspects of string theory and $M$-theory.

The extension of the ideas in this paper to higher order terms in the derivative expansion raises very interesting new issues, specially since the next term, of order $\partial^{8} \mathcal{R}^{4}$, is no longer expected to be BPS so there is a strong possibility that its coefficient, $\mathcal{E}_{(2,0)}^{(D)}$, gets contributions from all orders in string perturbation theory [5]. Although there is some information about this function based on its $M$-theory limit in $D=9$ [17], this is far from complete. Beyond that, it is not at all clear how the discrete symmetry acts on the complete scattering amplitude. Another clear challenge is the extension of these considerations to the coefficients in the low-energy expansion of multiparticle scattering amplitudes. Further afield are possible generalizations to amplitudes with non-maximal supersymmetry, which have lower-rank duality groups, or to scattering in curved space.

\section{Acknowledgments}

We would like to thank Ling Bao, Lisa Carbone, Pierre Cartier, Freydoon Shahidi, Laurent Lafforgue, Erez Lapid, Peter Sarnak and Wilfried Schmid for discussions. S.M. acknowledges support from grant DMS-0901594, and J.R. acknowledges support by MCYT Research Grant No. FPA 2007-66665 and Generalitat de Catalunya under project 2009SGR502. MBG is grateful for the support of a European Research Council Advanced Grant No. 247252.

Note: As this paper was being written a new appendix to [16] appeared making use of [26] and [5] to obtain results related to limit (i) of this paper. These results appear to be related to those presented here upon making use of identities between different maximal parabolic Eisenstein series such as those in (3.8). As explained at the end of section 2.3, the complete analysis performed in this paper requires properties of Eisenstein series that cannot be obtained by the method used in [26]. Other recent papers [37] also cover related topics. 


\section{Appendix A. Tables A.1 to A.8}

\section{A.1. Duality groups and maximal parabolic subgroups}

Table A.1: The moduli parameterize the coset $E_{d+1}(\mathbb{Z}) \backslash E_{d+1}(\mathbb{R}) / K$.

\begin{tabular}{lcccc}
\hline$D$ & Rank & $E_{d+1}(\mathbb{R})$ & $K$ & $E_{d+1}(\mathbb{Z})$ \\
\hline $10 \mathrm{~A}$ & 1 & $\mathbb{R}^{+}$ & 1 & 1 \\
$10 \mathrm{~B}$ & 1 & $\mathrm{SL}(2, \mathbb{R})$ & $\mathrm{SO}(2)$ & $\mathrm{SL}(2, \mathbb{Z})$ \\
9 & 2 & $\mathrm{SL}(2, \mathbb{R}) \times \mathbb{R}^{+}$ & $\mathrm{SO}(2)$ & $\mathrm{SL}(2, \mathbb{Z})$ \\
8 & 3 & $\mathrm{SL}(3, \mathbb{R}) \times \mathrm{SL}(2, \mathbb{R})$ & $\mathrm{SO}(3) \times \mathrm{SO}(2)$ & $\mathrm{SL}(3, \mathbb{Z}) \times \mathrm{SL}(2, \mathbb{Z})$ \\
7 & 4 & $\mathrm{SL}(5, \mathbb{R})$ & $\mathrm{SO}(5)$ & $\mathrm{SL}(5, \mathbb{Z})$ \\
6 & 5 & $\mathrm{SO}(5,5, \mathbb{R})$ & $\mathrm{SO}(5) \times \mathrm{SO}(5)$ & $\mathrm{SO}(5,5, \mathbb{Z})$ \\
5 & 6 & $E_{6(6)}(\mathbb{R})$ & $U S p(8)$ & $E_{6(6)}(\mathbb{Z})$ \\
4 & 7 & $E_{7(7)}(\mathbb{R})$ & $S U(8)$ & $E_{7(7)}(\mathbb{Z})$ \\
3 & 8 & $E_{8(8)}(\mathbb{R})$ & $\mathrm{SO}(16)$ & $E_{8(8)}(\mathbb{Z})$ \\
\hline
\end{tabular}

Table A.2: The parabolic subgroups associated with the simple roots $\alpha_{d+1}$, $\alpha_{1}$ and $\alpha_{2}$ of $E_{d+1}$.

\begin{tabular}{lccc}
\hline Deleted node & $E_{8}$ & $E_{7}$ & $E_{6}$ \\
\hline Right & $E_{7}$ & $E_{6}$ & $\mathrm{SO}(5,5)$ \\
Left & $\mathrm{SO}(7,7)$ & $\mathrm{SO}(6,6)$ & $\mathrm{SO}(5,5)$ \\
Upper & $\mathrm{SL}(8)$ & $\mathrm{SL}(7)$ & $\mathrm{SL}(6)$ \\
\hline \hline Deleted node & $E_{5}=\mathrm{SO}(5,5)$ & $E_{4}=\mathrm{SL}(5)$ & $E_{3}=\mathrm{SL}(3) \times \mathrm{SL}(2)$ \\
\hline Right & $\mathrm{SL}(5)$ & $\mathrm{SL}(3) \times \mathrm{SL}(2)$ & $\mathrm{SL}(2) \times \mathrm{SL}(2)$ \\
Left & $\mathrm{SO}(4,4)$ & $\mathrm{SO}(3,3)$ & $\mathrm{SO}(2,2)$ \\
Upper & $\mathrm{SL}(5)$ & $\mathrm{SL}(4)$ & $\mathrm{SL}(3)$ \\
\hline
\end{tabular}




\section{A.2. Solutions for $\mathcal{E}_{(0,0)}^{(D)}$ and $\mathcal{E}_{(1,0)}^{(D)}$ for rank- $(10-D)$ duality groups}

Table A.3: Solutions for the coefficients in $D=10-d$ dimensions.

\begin{tabular}{lcc}
\hline$G_{d}(\mathbb{Z})=E_{d+1}(\mathbb{Z})$ & $\mathcal{E}_{(0,0)}^{(D)}$ & $\mathcal{E}_{(1,0)}^{(D)}$ \\
\hline$E_{8(8)}(\mathbb{Z})$ & $\mathbf{E}_{\left[10^{7}\right] ; \frac{3}{2}}^{E_{8}}$ & $\frac{1}{2} \mathbf{E}_{\left[10^{7}\right] ; \frac{5}{2}}^{E_{8}}$ \\
$E_{7(7)}(\mathbb{Z})$ & $\mathbf{E}_{\left[10^{6}\right] ; \frac{3}{2}}^{E_{7}}$ & $\frac{1}{2} \mathbf{E}_{\left[10^{6}\right] ; \frac{5}{2}}^{E_{7}}$ \\
$E_{6(6)}(\mathbb{Z})$ & $\mathbf{E}_{\left[10^{5}\right] ; \frac{3}{2}}^{E_{6}}$ & $\frac{1}{2} \mathbf{E}_{\left[10^{5}\right] ; \frac{5}{2}}^{E_{6}}$ \\
$\mathrm{SO}(5,5, \mathbb{Z})$ & $\mathbf{E}_{[10000] ; \frac{3}{2}}^{\mathrm{SO}(5,5)}$ & $\frac{1}{2} \hat{\mathbf{E}}_{[10000] ; \frac{5}{2}}^{\mathrm{SO}(5,5)}+\frac{4}{45} \hat{\mathbf{E}}_{[00001] ; 3}^{\mathrm{SO}(5,5)}$ \\
$\mathrm{SL}(5, \mathbb{Z})$ & $\mathbf{E}_{[1000] ; \frac{3}{2}}^{\mathrm{SL}(5)}$ & $\frac{1}{2} \hat{\mathbf{E}}_{[1000] ; \frac{5}{2}}^{\mathrm{SL}(5)}+\frac{3}{\pi^{3}} \hat{\mathbf{E}}_{[0010] ; \frac{5}{2}}^{\mathrm{SL}(5)}$ \\
$\mathrm{SL}(3, \mathbb{Z}) \times \mathrm{SL}(2, \mathbb{Z})$ & $\hat{\mathbf{E}}_{[10] ; \frac{3}{2}}^{\mathrm{SL}(3)}+2 \hat{\mathbf{E}}_{1}(U)$ & $\frac{1}{2} \mathbf{E}_{[10] ; \frac{5}{2}}^{\mathrm{SL}(3)}-4 \mathbf{E}_{[10] ;-\frac{1}{2}}^{\mathrm{SL}(3)} \mathbf{E}_{2}(U)$ \\
$\mathrm{SL}(2, \mathbb{Z})$ & $\mathbf{E}_{\frac{3}{2}}(\Omega) \nu_{1}^{-\frac{3}{7}}+4 \zeta(2) \nu_{1}^{\frac{4}{7}}$ & $\frac{1}{2} \nu_{1}^{-\frac{5}{7}} \mathbf{E}_{\frac{5}{2}}(\Omega)+\frac{2 \zeta(2)}{15} \nu_{1}^{\frac{9}{7}}$ \\
$\mathrm{SL}(2, \mathbb{Z})$ & $\mathbf{E}_{\frac{3}{2}}(\Omega)$ & $\frac{1}{2} \mathbf{E}_{\frac{5}{2}}(\Omega)$ \\
\hline
\end{tabular}

The variables $\nu_{1}$ and $\Omega$ parameterize the GL(1) and $\mathrm{SL}(2) / \mathrm{SO}(2)$ factors in the $D=9$ moduli space. 


\section{A.3. Constant terms for $\mathcal{E}_{(0,0)}^{(D)}$ and $\mathcal{E}_{(1,0)}^{(D)}$}

Table A.4: The constant terms of $\mathcal{E}_{(0,0)}^{(D)}$ in the parabolic subgroups specified by limits (i) and (ii) for $D=3, \ldots, 9$.

\begin{tabular}{lcc}
\hline & $\begin{array}{c}\text { Decompactification limit } \\
\text { Constant term in } P_{\alpha_{d+1}}\end{array}$ & $\begin{array}{c}\text { String perturbation limit } \\
\text { Constant term in } P_{\alpha_{1}}\end{array}$ \\
$\mathcal{E}_{(0,0)}^{(D)}$ & $r^{2}=r_{d} / \ell_{D+1}$ & $r^{-4}=y_{D}$ \\
\hline $\mathbf{E}_{\left[10^{7}\right] ; \frac{3}{2}}^{E_{2}}$ & $\frac{3 \zeta(5)}{\pi} r^{20}+r^{12} \mathbf{E}_{\left[10^{6}\right] ; \frac{3}{2}}^{E_{7}}$ & $2 \zeta(3) r^{24}+r^{20} \frac{3}{2 \pi} \mathbf{E}_{\left[10^{6}\right] ; \frac{5}{2}}^{\mathrm{SO}(7)}$ \\
$\mathbf{E}_{\left[10^{6}\right] ; \frac{3}{2}}^{E_{7}}$ & $\frac{4 \zeta(4)}{\pi} r^{12}+r^{6} \mathbf{E}_{[10000] ; \frac{3}{2}}^{E_{6}}$ & $2 \zeta(3) r^{12}+r^{8} \frac{2}{\pi} \mathbf{E}_{[100000] ; 2}^{\mathrm{SO}(6,6)}$ \\
$\mathbf{E}_{\left[10^{5}\right] ; \frac{3}{2}}^{E_{6}}$ & $2 \zeta(3) r^{8}+r^{4} \mathbf{E}_{[10000] ; \frac{3}{2}}^{\mathrm{SO}(5,5)}$ & $2 \zeta(3) r^{8}+r^{4} \mathbf{E}_{[10000] ; \frac{3}{2}}^{\mathrm{SO}(5,5)}$ \\
$\mathbf{E}_{[10000] ; \frac{3}{2}}^{\mathrm{SO}(5,5)}$ & $4 \zeta(2) r^{5}+r^{3} \mathbf{E}_{[1000] ; \frac{3}{2}}^{\mathrm{SL}(5)}$ & $2 \zeta(3) r^{6}+2 r^{2} \mathbf{E}_{[1000] ; 1}^{\mathrm{SO}(4,4)}$ \\
$\mathbf{E}_{[1000] ; \frac{3}{2}}^{\mathrm{SL}(5)}$ & $r^{\frac{12}{5}}\left(\hat{\mathbf{E}}_{[10] ; \frac{3}{2}}^{\mathrm{SL}(3)}+2 \hat{\mathbf{E}}_{1}^{\mathrm{SL}(2)}\right.$ & $2 \zeta(3) r^{\frac{24}{5}}+2 \pi r^{\frac{4}{5}} \mathbf{E}_{[100] ; \frac{1}{2}}^{\mathrm{SO}(3,3)}$ \\
$\hat{\mathbf{E}}_{[10] ; \frac{3}{2}}^{\mathrm{SL}(3)}+2 \hat{\mathbf{E}}_{1}^{\mathrm{SL}(2)}$ & $-8 \pi \log r)$ & \\
$\nu_{1}^{-\frac{3}{7}} \mathbf{E}_{\frac{3}{2}}(\Omega)+4 \zeta(2) \nu_{1}^{\frac{4}{7}}$ & $4 \zeta(2) r^{-\frac{16}{7}}+r^{\frac{12}{7}} \mathbf{E}_{\frac{3}{2}}^{\mathrm{SL}(2)}$ & $2 \zeta(3) r^{\frac{24}{7}}+r^{-\frac{4}{7}} \mathbf{E}_{-\frac{1}{2}}^{\mathrm{SO}(1,1)}$ \\
& $r^{2}\left(\nu_{1}^{-\frac{3}{7}} \mathbf{E}_{\frac{3}{2}}^{\mathrm{SL}(2)}+4 \zeta(2) \nu_{1}^{\frac{4}{7}}\right)$ & $2 \zeta(3) r^{4}+2 \mathbf{E}_{[10] ; 0}^{\mathrm{SO}(2,2)}$ \\
& $-\frac{24 \pi}{3} \log r$ & $-\frac{8 \pi}{3} \log r$
\end{tabular}

The scales in the logarithms have been absorbed into the nonanalytic part of the string amplitude. 
Table A.5: The constant terms of $\mathcal{E}_{(0,0)}^{(D)}$ in the parabolic subgroups specified by limit (iii) for $D=3, \ldots, 9$.

\begin{tabular}{|c|c|}
\hline $\mathcal{E}_{(0,0)}^{(D)}$ & $\begin{array}{c}M \text {-theory limit Constant term in } \\
\text { Constant term in } P_{\alpha_{2}} \\
r^{(2+2 d) / 3}=\mathcal{V}_{d+1} / \ell_{11}^{d+1}\end{array}$ \\
\hline $\mathbf{E}_{\left[10^{7}\right] ; \frac{3}{2}}^{E_{8}}$ & $4 \zeta(2) r^{32}+r^{30} \mathbf{E}_{\left[10^{6}\right] ; \frac{3}{2}}^{\mathrm{SL}(8)}$ \\
\hline $\mathbf{E}_{\left[10^{6}\right] ; \frac{3}{2}}^{E_{7}}$ & $4 \zeta(2) r^{14}+r^{12} \mathbf{E}_{\left[10^{5}\right] ; \frac{3}{2}}^{\mathrm{SL}(7)}$ \\
\hline $\mathbf{E}_{\left[10^{5}\right] ; \frac{3}{2}}^{E_{6}}$ & $4 \zeta(2) r^{8}+r^{6} \mathbf{E}_{[10000] ; \frac{3}{2}}^{\mathrm{SL}(6)}$ \\
\hline $\mathbf{E}_{[10000] ; \frac{3}{2}}^{\mathrm{SO}(5,5)}$ & $4 \zeta(2) r^{5}+r^{3} \mathbf{E}_{[1000] ; \frac{3}{2}}^{\mathrm{SL}(5)}$ \\
\hline $\mathbf{E}_{[1000] ; \frac{3}{2}}^{\mathrm{SL}(5)}$ & $4 \zeta(2) r^{\frac{16}{5}}+r^{\frac{6}{5}} \mathbf{E}_{[100] ; \frac{3}{2}}^{\mathrm{SL}(4)}$ \\
\hline$\hat{\mathbf{E}}_{[10] ; \frac{3}{2}}^{\mathrm{SL}(3)}+2 \hat{\mathbf{E}}_{1}^{\mathrm{SL}(2)}$ & $4 \zeta(2) r^{2}+\hat{\mathbf{E}}_{[10] ; \frac{3}{2}}^{\mathrm{SL}(3)}-4 \pi \log r$ \\
\hline$\nu_{1}^{-\frac{3}{7}} \mathbf{E}_{\frac{3}{2}}(\Omega)+4 \zeta(2) \nu_{1}^{\frac{4}{7}}$ & $4 \zeta(2) r^{\frac{8}{7}}+r^{-\frac{6}{7}} \mathbf{E}_{\frac{3}{2}}^{\mathrm{SL}(2)}$ \\
\hline
\end{tabular}

The scale in the logarithm has again been absorbed into the nonanalytic part of the string amplitude. 
Table A.6: The constant terms of $\mathcal{E}_{(1,0)}^{(D)}$ in the parabolic subgroups specified by limit (i) in dimensions $D=3,4,5,6$.

\begin{tabular}{|c|c|}
\hline $\mathcal{E}_{(1,0)}^{(D)}$ & $\begin{array}{l}\text { Decompactification limit } \\
\text { Constant term in } P_{\alpha_{d+1}} \\
r^{2}=r_{d} / \ell_{D+1}\end{array}$ \\
\hline$\frac{1}{2} \mathbf{E}_{\left[10^{7}\right] ; \frac{5}{2}}^{E_{8}}$ & $\frac{1}{2} r^{20} \mathbf{E}_{\left[10^{6}\right] ; \frac{5}{2}}^{E_{7}}+\frac{\zeta(3)}{\pi} r^{24} \mathbf{E}_{\left[10^{6}\right] ; \frac{3}{2}}^{E_{7}}+\frac{7 \zeta(9)}{12 \pi} r^{36}$ \\
\hline$\frac{1}{2} \mathbf{E}_{\left[10^{6}\right] ; \frac{5}{2}}^{E_{7}}$ & $\frac{1}{2} r^{10} \mathbf{E}_{\left[10^{5}\right] ; \frac{5}{2}}^{E_{6}}+\frac{\pi}{3} r^{12} \mathbf{E}_{\left[10^{5}\right] ; \frac{3}{2}}^{E_{6}}+\zeta(5) r^{24}$ \\
\hline$\frac{1}{2} \mathbf{E}_{\left[10^{5}\right] ; \frac{5}{2}}^{E_{6}}$ & $r^{\frac{20}{3}}\left(\frac{1}{2} \hat{\mathbf{E}}_{[10000] ; \frac{5}{2}}^{\mathrm{SO}(5,5)}+\frac{4}{45} \hat{\mathbf{E}}_{[00001] ; 3}^{\mathrm{SO}(5,5)}\right)$ \\
\hline \multirow{3}{*}{$\frac{1}{2} \hat{\mathbf{E}}_{[10000] ; \frac{5}{2}}^{\mathrm{SO}(5,5)}+\frac{4}{45} \hat{\mathbf{E}}_{[00001] ; 3}^{\mathrm{SO}(5,5)}$} & $+r^{\frac{20}{3}} \log r \mathbf{E}_{[10000] ; \frac{3}{2}}^{\mathrm{SO}(5,5)}+\frac{\zeta(7)}{6} r^{\frac{56}{3}}$ \\
\hline & $\frac{4 \zeta(5)}{45} r^{9}+r^{5} \log r \mathbf{E}_{[1000] ; \frac{3}{2}}^{\mathrm{SL}(5)}$ \\
\hline & $+r^{3}\left(\frac{1}{2} \hat{\mathbf{E}}_{[1000] ; \frac{5}{2}}^{\mathrm{SL}(5)}+\frac{3}{\pi^{2}} \hat{\mathbf{E}}_{[0010] ; \frac{5}{2}}^{\mathrm{SL}(5)}\right.$ \\
\hline
\end{tabular}

The scale in the logarithm has again been absorbed into the nonanalytic part of the string amplitude.

Table A.7: The constant terms of $\mathcal{E}_{(1,0)}^{(D)}$ in the parabolic subgroups specified by limit (ii) in dimensions $D=3,4,5,6$.

\begin{tabular}{|c|c|}
\hline $\mathcal{E}_{(1,0)}^{(D)}$ & $\begin{array}{l}\text { String perturbation limit } \\
\text { Constant term in } P_{\alpha_{1}} \\
r^{-4}=y_{D}\end{array}$ \\
\hline$\frac{1}{2} \mathbf{E}_{\left[10^{7}\right] ; \frac{5}{2}}^{E_{8}}$ & $r^{40} \zeta(5)+\frac{7}{24 \pi} r^{36} \mathbf{E}_{\left[10^{6}\right] ; \frac{9}{2}}^{\mathrm{SO}(7,7)}+\frac{2}{3} r^{32} \mathbf{E}_{\left[0^{5} 10\right] ; 2}^{\mathrm{SO}(7,7)}$ \\
\hline$\frac{1}{2} \mathbf{E}_{\left[10^{6}\right] ; \frac{5}{2}}^{E_{7}}$ & $r^{20} \zeta(5)+\frac{4}{15 \pi} r^{16} \mathbf{E}_{\left[10^{5}\right] ; 4}^{\mathrm{SO}(6,6)}+\frac{2}{3} r^{12} \mathbf{E}_{\left[0^{4} 10\right] ; 2}^{\mathrm{SO}(6,6)}$ \\
\hline$\frac{1}{2} \mathbf{E}_{\left[10^{5}\right] ; \frac{5}{2}}^{E_{6}}$ & $r^{\frac{40}{3}} \zeta(5)+\frac{1}{12} r^{\frac{28}{3}} \mathbf{E}_{[10000] ; \frac{7}{2}}^{\mathrm{SO}(5,5)}+\frac{2}{3} r^{\frac{16}{3}} \mathbf{E}_{[00010] ; 2}^{\mathrm{SO}(5,5)}$ \\
\hline$\frac{1}{2} \hat{\mathbf{E}}_{[10000] ; \frac{5}{2}}^{\mathrm{SO}(5,5)}$ & $r^{10} \zeta(5)+r^{2} \mathbf{E}_{[1000] ; 3}^{\mathrm{SO}(4,4)}+\frac{2}{3} r^{6}\left(\mathbf{E}_{[0010] ; 2}^{\mathrm{SO}(4,4)}+\mathbf{E}_{[0001] ; 2}^{\mathrm{SO}(4,4)}\right)$ \\
\hline$+\frac{4}{45} \hat{\mathbf{E}}_{[00001] ; 3}^{\mathrm{SO}(5,5)}$ & \\
\hline
\end{tabular}


Table A.8: The constant terms of $\mathcal{E}_{(1,0)}^{(D)}$ in the parabolic subgroups specified by limit (iii) in dimensions $D=3,4,5,6$.

\begin{tabular}{lc}
\hline & $\begin{array}{r}M \text {-theory limit Constant term in } \\
\text { Constant term in } P_{\alpha_{2}} \\
r^{(2+2 d) / 3}=\mathcal{V}_{d+1} / \ell_{11}^{d+1}\end{array}$ \\
$\mathcal{E}_{(1,0)}^{(D)}$ & $\frac{1}{2} r^{50} \mathbf{E}_{\left[10^{6}\right] ; \frac{5}{2}}^{\mathrm{SL}(8)}+\frac{2 \zeta(3)}{\pi^{2}} r^{48} \mathbf{E}_{\left[010^{5}\right] ; 2}^{\mathrm{SL}(8)}-8 \zeta(4) r^{54} \mathbf{E}_{\left[10^{6}\right] ;-\frac{1}{2}}^{\mathrm{SL}(8)}$ \\
$\frac{1}{2} \mathbf{E}_{\left[10^{7}\right] ; \frac{5}{2}}^{E_{8}}$ & $\frac{1}{2} r^{20} \mathbf{E}_{\left[10^{5}\right] ; \frac{5}{2}}^{\mathrm{SL}(7)}+\frac{2 \zeta(3)}{\pi^{2}} r^{18} \mathbf{E}_{\left[010^{5}\right] ; 2}^{\mathrm{SL}(8)}-8 \zeta(4) r^{24} \mathbf{E}_{\left[10^{5}\right] ;-\frac{1}{2}}^{\mathrm{SL}(7)}$ \\
$\frac{1}{2} \mathbf{E}_{\left[10^{6}\right] ; \frac{5}{2}}^{E_{7}}$ & $\frac{1}{2} r^{10} \mathbf{E}_{\left[10^{4}\right] ; \frac{5}{2}}^{\mathrm{SL}(6)}+\frac{2 \zeta(3)}{\pi^{2}} r^{8} \mathbf{E}_{\left[010^{3}\right] ; 2}^{\mathrm{SL}(6)}-8 \zeta(4) r^{14} \mathbf{E}_{\left[10^{4}\right] ;-\frac{1}{2}}^{\mathrm{SL}(6)}$ \\
$\frac{1}{2} \mathbf{E}_{\left[10^{5}\right] ; \frac{5}{2}}^{E_{6}}$ & $\frac{4 \zeta(5)}{45} r^{9}+r^{5} \log r \mathbf{E}_{[1000] ; \frac{3}{2}}^{\mathrm{SL}(5)}+r^{3}\left(\frac{1}{2} \hat{\mathbf{E}}_{[1000] ; \frac{5}{2}}^{\mathrm{SL}(5)}+\frac{3}{\pi^{2}} \hat{\mathbf{E}}_{[0010] ; \frac{5}{2}}^{\mathrm{SL}(5)}\right.$ \\
$\frac{1}{2} \hat{\mathbf{E}}_{[10000] ; \frac{5}{2}}^{\mathrm{SO}(5,5)}$ & \\
$+\frac{4}{45} \hat{\mathbf{E}}_{[00001] ; 3}^{\mathrm{SO}(5,5)}$ & \\
\hline
\end{tabular}

The scales in the logarithms have again been absorbed into the nonanalytic part of the string amplitude.

\section{References}

[1] C. M. Hull and P. K. Townsend, Unity of superstring dualities, Nucl. Phys. B438 (1995), 109 [arXiv:hep-th/9410167].

[2] C. Chevalley, Certains schémas de groupes semi-simples, Séminaire Bourbaki, exposé 219 (1960), 1.

[3] M. B. Green, J. H. Schwarz and E. Witten, Superstring theory, Cambridge University Press, Cambridge, 1987 (Cambridge Monographs On Mathematical Physics)

[4] M. B. Green, J. G. Russo and P. Vanhove, Automorphic properties of low energy string amplitudes in various dimensions, arXiv:1001.2535 [hep-th].

[5] M. B. Green, J. G. Russo and P. Vanhove, String theory dualities and supergravity divergences, JHEP 1006 (2010), 075 [arXiv:1002.3805 [hep-th]].

[6] M. B. Green and M. Gutperle, Effects of D-instantons, Nucl. Phys. B498 (1997), 195 [arXiv:hep-th/9701093]. 
[7] M. B. Green, M. Gutperle and P. Vanhove, One loop in eleven dimensions, Phys. Lett. B409 (1997), 177 [arXiv:hep-th/9706175].

[8] J. G. Russo and A. A. Tseytlin, One-loop four-graviton amplitude in eleven-dimensional supergravity, Nucl. Phys. B508 (1997), 245 [arXiv:hep-th/9707134].

[9] M. B. Green and P. Vanhove, Duality and higher derivative terms in $M$ theory, JHEP 0601 (2006), 093 [arXiv:hep-th/0510027].

[10] E. Kiritsis and B. Pioline, On $R^{4}$ threshold corrections in type IIB string theory and $(p, q)$ string instantons, Nucl. Phys. B508 (1997), 509 [arXiv:hep-th/9707018].

[11] A. Basu, The $D^{4} R^{4}$ term in type IIB string theory on $T^{2}$ and U-duality, Phys. Rev. D77 (2008), 106003 [arXiv:0708.2950 [hep-th]].

[12] A. Basu, The $\partial^{6} R^{4}$ term in type IIB string theory on $T^{2}$ and $U$-duality, arXiv:0712.1252 [hep-th].

[13] N. A. Obers and B. Pioline, Eisenstein series and string thresholds, Commun. Math. Phys. 209 (2000), 275 [arXiv:hep-th/9903113].

[14] L. Bao, M. Cederwall and B. E. W. Nilsson, Aspects of higher curvature terms and U-duality, Class. Quant. Grav. 25 (2008), 095001 [arXiv:0706.1183 [hep-th]]; L. Bao, J. Bielecki, M. Cederwall, B. E. W. Nilsson and D. Persson, U-duality and the compactified Gauss-Bonnet term, JHEP 0807 (2008), 048 [arXiv:0710.4907 [hep-th]].

[15] N. Lambert and P. C. West, Duality groups, automorphic forms and higher derivative corrections, Phys. Rev. D75 (2007), 066002 [arXiv:hep-th/0611318]; N. D. Lambert and P. C. West, Coset symmetries in dimensionally reduced bosonic string theory, Nucl. Phys. B615 (2001), 117 [arXiv:hep-th/0107209].

[16] B. Pioline, $R^{4}$ couplings and automorphic unipotent representations, arXiv:1001.3647 [hep-th].

[17] M. B. Green, J. G. Russo and P. Vanhove, Modular properties of twoloop maximal supergravity and connections with string theory, JHEP 0807 (2008), 126 [arXiv:0807.0389 [hep-th]].

[18] M. B. Green and S. Sethi, Supersymmetry constraints on type IIB supergravity, Phys. Rev. D59 (1999), 046006 [arXiv:hep-th/ 9808061]. 
[19] S. D. Miller, Spectral and cohomological applications of the RankinSelberg method, Int. Math. Res. Notices (1), (1996) 15-26.

[20] S. D. Miller, The highest-lowest zero and other applications of positivity, Duke Math. J. 112(1), 83-116.

[21] R. Langlands, On the functional equations satisfied by Eisenstein series, Lecture Notes in Mathematics, 544, Springer, Berlin, 1976.

[22] R. Langlands, Euler products, Yale Mathematical Monographs, 1, Yale University Press, 1971.

[23] S. Mizoguchi and G. Schroder, On discrete U-duality in M-theory, Class. Quant. Grav. 17 (2000), 835 [arXiv:hep-th/9909150].

[24] A. Knapp, Lie groups, lie algebras, and cohomology, Princeton Mathematical Notes, Princeton University Press, 1988.

[25] C. Moeglin and J. L. Waldspurger, Spectral decomposition and Eisenstein series: a paraphrase of the scriptures, Cambridge Tracts in Mathematics, July 31, 2008.

[26] D. Ginzburg, S. Ralli, and D. Soudry, On the automorphic theta representation for simply laced groups, Israel J. Math. 100 (1997), 61-116.

[27] S. S. Kudla and S. Rallis, Poles of Eisenstein series and L-functions, in 'Festschrift in honor of I. I. Piatetski-Shapiro on the occasion of his sixtieth birthday, Part II' (Ramat Aviv, 1989), Israel Math. Conf. Proc., 3, 81-110, Weizmann, Jerusalem, 1990.

[28] M. B. Green, J. G. Russo and P. Vanhove, Non-renormalisation conditions in type II string theory and maximal supergravity, JHEP 0702, (2007), 099 [arXiv:hep-th/0610299].

[29] M. B. Green and P. Vanhove, The Low energy expansion of the oneloop type II superstring amplitude, Phys. Rev. D61 (2000), 104011 [arXiv:hep-th/9910056].

[30] M. B. Green, J. G. Russo and P. Vanhove, Low energy expansion of the four-particle genus-one amplitude in type II superstring theory, JHEP 0802 (2008), 020 [arXiv:0801.0322 [hep-th]].

[31] M. B. Green, H. h. Kwon and P. Vanhove, Two loops in eleven dimensions, Phys. Rev. D61 (2000), 104010 [arXiv:hep-th/9910055].

[32] A. Weil, Sur la formule de Siegel dans la theorie des groupes classiques, Acta Math. 113 (1965), 1-87. 
[33] C. L. Siegel, Symplectic geometry, Amer. J. Math. 65 (1943), 1-86; Academic Press, New York and London, 1964; Gesammelte Abhandlungen, no. 41, vol. II, Springer-Verlag, 1966, 274-359.

[34] M. B. Green and M. Gutperle, D-Instanton partition functions, Phys. Rev. D58 (1998), 046007 [arXiv:hep-th/9804123].

[35] G. W. Moore, N. Nekrasov and S. Shatashvili, Commun. Math. Phys. 209 (2000), 77 [arXiv:hep-th/9803265].

[36] F. Sugino and P. Vanhove, U-duality from matrix membrane partition function, Phys. Lett. B522 (2001), 145 [arXiv:hep-th/0107145].

[37] N. Lambert and P. West, Perturbation theory from automorphic forms, arXiv:1001.3284 [hep-th]; F. Gubay, N. Lambert and P. West, Constraints on automorphic forms of higher derivative terms from compactification, arXiv:1002.1068 [hep-th].

Department of Applied Mathematics and Theoretical Physics

WILBERForCE ROAD

CAmbridge CB3 0WA, UK

E-mail address: M.B.Green@damtp.cam.ac.uk

Department of Mathematics

RUtGers UNIVERSITY

Piscataway

NJ 08854-8019, USA

E-mail address: miller@math.rutgers.edu

Institució Catalana de Recerca i Estudis Avançats (ICREA)

Departament ECM and Institut de Ciencies del Cosmos

UNIVERSITY DE BARCELONA

Facultat de Fisica Av. Diagonal, 647

BARCElona 08028, SPAin

E-mail address: jrusso@ub.edu

Institut des Hautes Etudes Scientifiques

Le Bois-Marie, 35 route de Chartres

F-91440 Bures-Sur-Yvette, France

Institut de Physique Théorique

CEA, IPнT

F-91191 Gif-Sur-Yvette, France

CNRS

URA 2306

F-91191 Gif-Sur-Yvette, France

E-mail address: pierre.vanhove@cea.fr

Received APRIL 19, 2010 